\title{
Instability Regimes and Self-Excited Vibrations in Deep Drilling Systems
}

\author{
Alexandre Depouhon ${ }^{\mathrm{a}}$, Emmanuel Detournay ${ }^{\mathrm{a}, \mathrm{b}}$ \\ ${ }^{a}$ Department of Civil Engineering, University of Minnesota, USA \\ ${ }^{b}$ Earth Science and Resource Engineering, CSIRO, Australia
}

\begin{abstract}
This paper analyzes the stability of the discrete model proposed by Richard et al. [1,2] to study the self-excited axial and torsional vibrations of deep drilling systems. This model, which relies on a rate-independent bit/rock interaction law, reduces to a coupled system of state-dependent delay differential equations governing the axial and angular perturbations to the stationary motion of the bit. A linear stability analysis indicates that, although the steady-state motion of the bit is always unstable, the nature of the instability depends on the nominal angular velocity $\Omega_{0}$ of the drillstring imposed at the rig. On the one hand, if $\Omega_{0}$ is larger than a critical velocity $\Omega_{c}$, the angular dynamics is responsible for the instability. However, on the timescale of the resonance period of the drillstring viewed as a torsional pendulum, the system behaves like a marginally stable one, provided that exogenous perturbations are of limited magnitude. The instability then only appears on a much larger timescale, in the form of slowly growing oscillations that ultimately lead to an undesired drilling regime such as bit-bouncing or stick-slip vibrations. On the other hand, if $\Omega_{0}$ is smaller than $\Omega_{c}$, the instability manifests itself on the timescale of the bit motion due to a dominating unstable axial dynamics; perturbations to the steady-state motion then rapidly degenerate into stick-slip limit cycles or bit-bouncing. For typical deep drilling field conditions, the critical angular velocity $\Omega_{c}$ is virtually independent of the axial force acting on the bit and of the bit bluntness. It can be approximated by a power law monomial, a function of known parameters of the drilling system and of the intrinsic specific energy (a quantity characterizing the energy required to drill a particular rock). This approximation holds on account that the dissipation in the drilling structure is negligible with respect to that taking place through the bit/rock interaction, as is typically the case. These findings are further illustrated on an example of deep drilling and shown to match the trends observed in the field.
\end{abstract}

Keywords: self-excited vibrations, drilling, stick-slip, stability analysis, state-dependent delay, delayed differential equations

\footnotetext{
${ }^{*}$ Department of Civil Engineering, 500 Pillsbury Drive SE, Minneapolis, MN 55455
}

Email address: detou001@umn.edu (Emmanuel Detournay) 


\section{Introduction}

Rotary drilling systems that are used to drill deep boreholes for hydrocarbon exploration and production often exhibit complex self-excited vibratory behaviors. In particular, according to down-hole measurements [3], drilling structures continually experience torsional vibrations. For drag bit equipped systems, these vibrations frequently degenerate into torsional stick-slip oscillations that are characterized by $(i)$ sticking phases during which the drill bit stops rotating, and (ii) slipping phases during which the tool sees its angular velocity increase up to two times the imposed velocity on the ground surface. Stick-slip oscillations are detrimental to the bit and the drillstring, as they are responsible for an accelerated wear of the drill bit and fatigue of the drill pipe connections. Their understanding is thus fundamental to the improvement of the drilling process. 0 The stick-slip vibrations experienced by rotary drilling systems are conventionally analyzed by modeling the drillstring as a torsional pendulum and the bit/rock interaction as a reactive torque that decreases with the bit angular velocity, in apparent accordance with measurements from field experiments [4-10]. However, an alternative approach to predict stick-slip oscillations of the bit was proposed by Richard et al. [1, 2]. This model, here referred to as the RGD model, relies instead on a rate-independent bit/rock interaction law that reflects not only the nature of the regenerative cutting and frictional contact processes taking place at the bit/rock interface [11], but that also introduces a coupling between the axial and angular motions of the bit.

Formulation of the RGD model leads to a coupled system of nonlinear state-dependent delay differential equations governing the axial and angular perturbations to the stationary motion of the bit, with the axial dynamics typically developing on a much smaller timescale than the torsional one, due mainly to the large torsional compliance of the drillstring. Interestingly, the RGD model predicts a decrease of the resisting torque under an imposed angular velocity at the rig; thus the velocity weakening response of the bit is an outcome of the RGD model, rather than a starting hypothesis as in the classical approach. Models built on the same rate-independent bit/rock interface law, but that include additional degrees of freedom and viscous dissipation in the representation of the drillstring and/or consider alternate boundary conditions at the rig, have recently been studied [12-15]; they yield similar although richer responses than the basic RGD model. Also, comparable state-dependent delay models to analyze regenerative chatter in metal turning processes have been independently developed by Insperger et al. [16-18].

The motivation to revisit the RGD model, in particular in regard to stability issues, stems from two apparently contradictory results. On the one hand, the RGD model is always unstable according to a recently published linear stability analysis [14]. Previous stability analyses [2,19] neglected the dynamics of the delay based on the assumption of a timescale separation between the axial and torsional dynamics, and concluded that the steady-state solution is unstable only if the prescribed angular velocity is below a critical velocity. Under these conditions, any perturbation will lead the system to diverge from its equilibrium solution, ultimately leading to bit-bouncing, stick-slip limit cycling or anti-resonance, a regime in which large amplitude axial vibrations of the bit stabilize its angular motion [19]. On the other hand, there is evidence from numerical simulations that quasiperiodic perturbations to the angular motion exist for particular parametric configurations [2, 19]. 


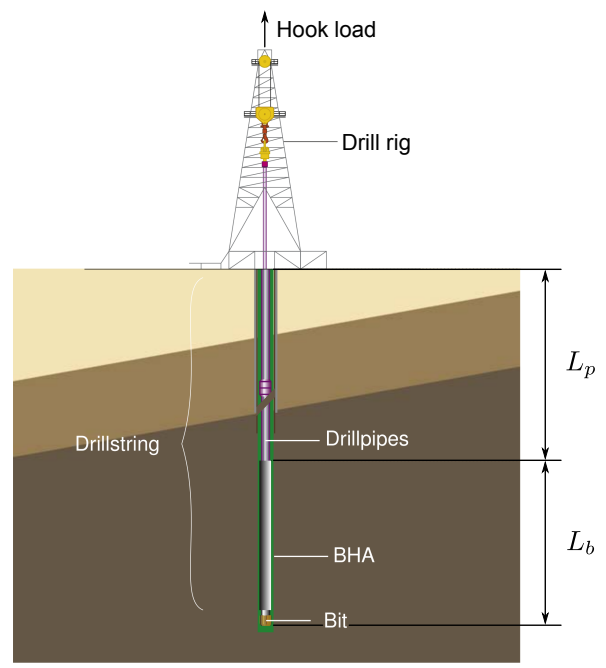

Figure 1: Deep drilling system, adapted from [20]. The sketch is not to scale, in particular, in regard to the length $L_{b}$ of the bottom-hole-assembly (BHA) relative to the combined length $L_{p}$ of the drill pipes. Typically, $L_{b}=O(100 \mathrm{~m})$ and $L_{p}=O(1000 \mathrm{~m})$.

This paper aims therefore at clarifying the nature of the instabilities in the RGD model of the drilling system. After a brief presentation of this two degree of freedom model [1, 2], we formulate the stability problem of the steadystate motion of the drill bit, taking into account the state dependency of the delay when linearizing the governing equations. We then confirm that the trivial motion is indeed always unstable, as reported in [14]. However, by studying the location of the linearized system poles in the complex plane, we prove the existence of two regimes of instability, on either side of a critical angular velocity, the nominal rotation speed at the rig at which the transition takes place. One regime (slow) corresponds to a very slow growth of torsional oscillations and the other (fast) to a fast increase of axial perturbations that rapidly lead to stick-slip torsional oscillations, bit bouncing or anti-resonance. For typical field conditions, the critical velocity is shown to be well approximated by the expression obtained by assuming that the delay is constant and neglecting the influence of the axial force on the bit (the so-called "weight on bit"). Further exploring the slow regime by use of transient numerical simulations, we demonstrate the possible coexistence of drilling responses; that is, the coexistence of apparently stable steady drilling and instabilities like stick-slip limit cycles or bit-bouncing, depending on the magnitude of the externally-applied perturbations. Finally, we illustrate our findings by application to a configuration typical of deep drilling and summarize them in the last section of this article. Extended results and details of calculations are also provided in the appendices.

\section{Mathematical formulation}

The RGD model is a discrete representation of a rotary drilling structure with boundary conditions at the rig and at the bit/rock interface. Figure 1 proposes an illustrative sketch of such a structure. 


\subsection{Model of the Drilling System}

A rotary drilling structure consists essentially of a rig, a drillstring, and a bit. The principal components of the drillstring are the bottom-hole-assembly (BHA) composed of heavy tubes, and a set of drill pipes made of thinner tubes. The lumped mechanical model of the drillstring consists of a torsional pendulum of polar mass moment of inertia $I$ and stiffness $C$, and a punctual mass $M$ free to move axially, see Figure 2(a). The mass $M$ and the moment of inertia $I$ are taken to represent the BHA, and the stiffness $C$ the set of drill pipes.

Denoting the weight and torque on bit by $W$ and $T$, the equations of motion read

$$
\begin{aligned}
I \frac{\mathrm{d}^{2} \Phi}{\mathrm{d} t^{2}}+C\left(\Phi-\Omega_{0} t\right) & =-T\left(U_{t}, \Phi_{t}\right), \\
M \frac{\mathrm{d}^{2} U}{\mathrm{~d} t^{2}} & =W_{0}-W\left(U_{t}, \Phi_{t}\right),
\end{aligned}
$$

where $\Phi$ and $U$ represent the angular and axial positions of the bit, $\Omega_{0}$ is the constant imposed angular velocity of the rig rotary table and $W_{0}=W_{s}-H_{0}$ is the apparent weight of the drillstring acting on the bit, i.e., the difference between the submerged weight $W_{s}$ of the drilling system and the prescribed hook load $H_{0}$ at the rig. Due to the regenerative cutting taking place at the bit/rock interface, the reacting weight and torque on bit depend on the history of the bit motion, as indicated by the subscript ${ }_{t}$ in $U_{t}$ and $\Phi_{t}$.Thus, equations (1)-(2) are actually functional differential equations.

(a)

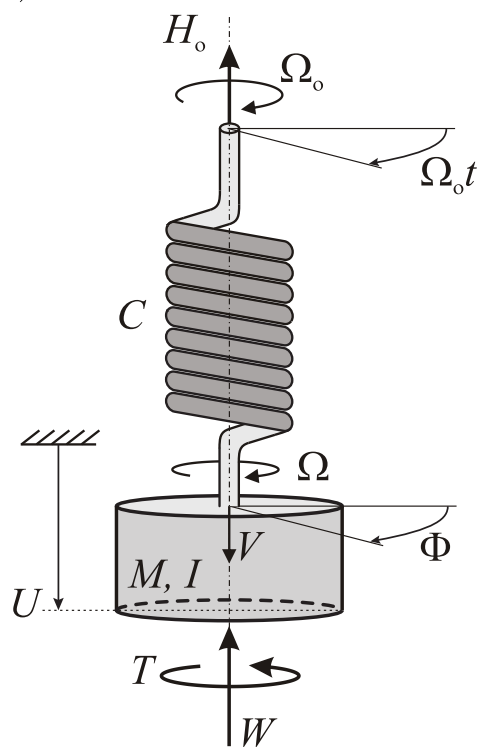

(b)

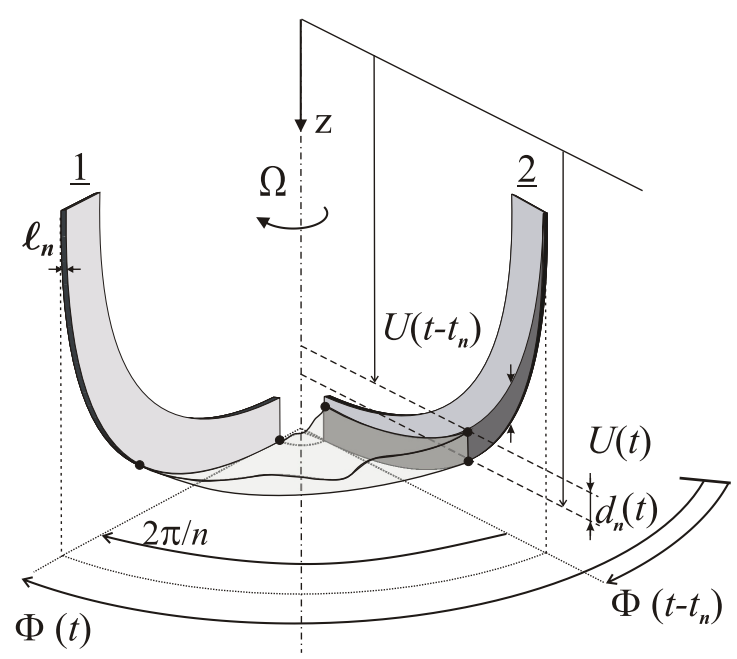

Figure 2: (a) Lumped model of the drilling system - (b) Section of the bottom-hole profile located between two successive blades of a drill bit, after [2]. 


\subsection{Bit/Rock Interaction Law}

The drilling action of a drag bit results from a pure cutting process taking place on the cutting face of the bit blades and from a frictional contact process at the interface between the rock and the cutter wear flats [11]. When drilling, the weight and torque on bit consist therefore of two components,

$$
W=W_{c}+W_{f}, \quad T=T_{c}+T_{f},
$$

where the subscripts ${ }_{c}$ and ${ }_{f}$ refer to the cutting and frictional contact processes.

Consider now the idealized bit of Figure 2(b), which is characterized by a radius $a$ and $n$ identical blades, symmetrically distributed around its axis of revolution. A bit/rock interaction law for such a bit, i.e., a set of relationships between the dynamical variables $W$ and $T$ and the kinematical variables $U_{t}$ and $\Phi_{t}$, can be constructed from experimental results obtained from single cutter tests [11,21, 22].

The cutting components of $W$ and $T$ are given by

$$
W_{c}(t)=a \zeta \varepsilon d(t), \quad T_{c}(t)=\frac{a^{2}}{2} \varepsilon d(t),
$$

where $\varepsilon$ is the intrinsic specific energy of the rock, namely the amount of energy required to remove a unit volume of rock by cutting, $\zeta$ characterizes the inclination of the force acting on the blade cutting face, and $d=n d_{n}$ is the bit depth of cut with $d_{n}$ the depth of cut per blade, i.e., the height of rock ahead of a blade. (For stationary bit motions, $d$ represents the depth of cut per revolution.) As the bit experiences axial and torsional vibrations when drilling, the depth of cut $d$ varies with time $t$. Due to the bit angular motion, regenerative cutting takes place and $d(t)$ depends on the past motion of the bit according to

$$
d(t)=n\left[U(t)-U\left(t-t_{n}(t)\right)\right],
$$

where the delay $t_{n}(t)$ is the time required for the bit to rotate by an angle of $2 \pi / n$ to its current position at time $t$. The delay $t_{n}(t)$ is thus implicitly defined by (see Figure 2(b))

$$
2 \pi=n\left[\Phi(t)-\Phi\left(t-t_{n}(t)\right)\right]
$$

Assuming that the bit is rotating forward $(\mathrm{d} \Phi / \mathrm{d} t>0)$, the expressions for the frictional contact components of $W$ and $T$ depend on the axial velocity $\mathrm{d} U / \mathrm{d} t$. If the bit moves downwards ( $\mathrm{d} U / \mathrm{d} t>0)$, a constant normal stress $\sigma$ and thus a constant shear stress $\mu \sigma$ act on the wear flat surfaces in contact with the rock, with $\mu$ denoting a rate-independent friction coefficient. Hence, when $\mathrm{d} U / \mathrm{d} t>0, W_{f}=a \ell \sigma$ and $T_{f}=\frac{1}{2} a^{2} \gamma \mu \ell \sigma$, where $\ell=n \ell_{n}$ with $\ell_{n}$ denoting the width of the contact surface underneath each blade, and $\gamma$ is the bit number that globally characterizes the spatial distribution and orientation of the wear flats along the bit profile [11]. If the bit moves upwards $(\mathrm{d} U / \mathrm{d} t<0)$, there is no frictional contact and thus $W_{f}=T_{f}=0$. Finally, the bit sticks axially $(\mathrm{d} U / \mathrm{d} t=0)$ as long as the contact force $W_{f}(t)$, then determined by the vertical equilibrium conditions, remains in the range $[0, a \ell \sigma]$, with the lower and upper limits respectively corresponding to the transition to upwards and downwards motion of the bit. Similarly, provided that $\mathrm{d} \Phi / \mathrm{d} t>0$, the frictional torque $T_{f}(t)$ that is proportional to $W_{f}(t)$ ranges in $\left[0, \frac{1}{2} a^{2} \gamma \mu \ell \sigma\right]$. 
Given their set-valued nature during axial stick, the frictional contact components of the weight and torque on bit can be elegantly represented via an inclusion formalism. Following Germay et al. [19], the above assumptions summarize as

$$
W_{f}(\mathrm{~d} U / \mathrm{d} t) \in \frac{1}{2} a \ell \sigma[1+\operatorname{Sign}(\mathrm{d} U / \mathrm{d} t)], \quad T_{f}(\mathrm{~d} U / \mathrm{d} t)=\frac{1}{2} \gamma \mu a W_{f}(\mathrm{~d} U / \mathrm{d} t)
$$

with the set-valued Sign operator defined by

$$
\operatorname{Sign}(x):=\partial|x| \in\left\{\begin{array}{cc}
1 & x>0 \\
{[-1,1]} & x=0 \\
-1 & x<0
\end{array}\right.
$$

These inclusions can be interpreted as the convexification of the contact force and torque for positive and negative velocities. Such convexified representations are common in mechanical problems involving friction. When considered at the level of the governing differential equation, they are referred to as Filippov inclusions. Leine and van de Wouw [23] provide an introduction to this formalism.

\subsection{Scaling}

The RGD model depends on a set of 13 parameters $\left(M, I, C, n, a, \varepsilon, \sigma, \zeta, \mu, \ell, \gamma, \Omega_{0}, W_{0}\right)$, which can be reduced through scaling to 6 dimensionless quantities $\left(n, \lambda, \beta, \psi, \omega_{0}, \mathcal{W}_{0}\right)$ [2]. First introduce a timescale $t_{*}$ and a length scale $d_{*}$ as

$$
t_{*}=\sqrt{\frac{I}{C}}, \quad d_{*}=\frac{2 C}{\varepsilon a^{2}},
$$

where $2 \pi t_{*}$ represents the resonant period of the torsional pendulum and $d_{*}$ is the depth of cut if drilling with a perfectly sharp bit subjected to a torque that induces a twist of 1 radian in the drillstring. Typically, $t_{*}=O(1 \mathrm{~s})$ and $d_{*}=O(1 \mathrm{~mm})$. The dimensionless time as well as the scaled kinematic and dynamical quantities at the bit can then be defined as

$$
\tau=\frac{t}{t_{*}}, \quad u=\frac{U-U_{0}}{d_{*}}, \quad v=\frac{t_{*}}{d_{*}} \frac{\mathrm{d} U}{\mathrm{~d} t}, \quad \varphi=\Phi-\Phi_{0}, \quad \omega=t_{*} \frac{\mathrm{d} \Phi}{\mathrm{d} t}, \quad W=\frac{W}{\zeta a \varepsilon d_{*}}, \quad \mathcal{T}=\frac{T}{C},
$$

where $U_{0}$ and $\Phi_{0}$ denote the stationary solutions of system (1)-(2) given by

$$
U_{0}(t)=V_{0} t=\frac{\left(W_{0}-W_{f}\left(V_{0}\right)\right)}{a \zeta \varepsilon} \frac{\Omega_{0}}{2 \pi} t, \quad \Phi_{0}=\Omega_{0} t-\frac{1}{C}\left(T_{f}\left(V_{0}\right)+\frac{a}{2 \zeta}\left(W_{0}-W_{f}\left(V_{0}\right)\right)\right) .
$$

The axial and angular displacements, $U_{0}$ and $\Phi_{0}$, correspond to steady drilling $\left(V_{0}>0\right)$ in the absence of vibrations, while $u$ and $\varphi$ represent perturbations of the axial and angular positions of the bit with respect to the stationary solution.

The six numbers defining the RGD model consist of two control variables, namely the rotation speed at the surface and the weight on bit,

$$
\omega_{0}=\Omega_{0} t_{*}, \quad W_{0}=\frac{W_{0}}{\zeta a \varepsilon d_{*}}
$$


the number of blades $n$, and three lumped parameters

$$
\beta=\mu \gamma \zeta, \quad \lambda=\frac{\sigma \ell}{\zeta \varepsilon d_{*}}, \quad \psi=\frac{\zeta \varepsilon a I}{M C}
$$

that respectively represent the bit geometry, the bit bluntness state and the design of the drillstring. Except for the system number $\psi$ that takes large values $O\left(10-10^{3}\right)$, the other lumped parameters and the control variables $\omega_{0}$ and $\mathcal{W}_{0}$ are $O(1)$. It is convenient, however, to replace $\omega_{0}$ and $\mathcal{W}_{0}$ by $\tau_{n 0}$ and $\alpha_{0}$, the surrogate control variables defined as

$$
\tau_{n 0}=\frac{2 \pi}{n \omega_{0}}, \quad \alpha_{0}=\frac{W_{0}-\lambda}{2 \pi} .
$$

The constant delay $\tau_{n 0}$ is the dimensionless time required to rotate the bit by the angle $2 \pi / n$ between two blades at steady state and $\alpha_{0}$ is a measure of the active weight on bit at steady state, i.e., the weight on bit minus the axial force transmitted by the bit wear flats. Since $\alpha_{0}=v_{0} / \omega_{0}, \alpha_{0}$ is proportional to the bit penetration per revolution while drilling in steady state; indeed, $\alpha_{0}=d_{0} / 2 \pi d_{*}$ with $d_{0}=2 \pi V_{0} / \Omega_{0}$. Both $\tau_{n 0}$ and $\alpha_{0}$ are typically $O(0.1)$.

After scaling, the RGD model can then be formulated as two coupled state-dependent delay differential inclusions governing the evolution of axial and angular perturbations $u(\tau)$ and $\varphi(\tau)$

$$
\begin{array}{r}
\ddot{u} \in n \psi\left\{\left[(\tilde{u}-u)-\alpha_{0}(\tilde{\varphi}-\varphi)\right]+\mathrm{g}(\dot{u})\right\}, \\
\ddot{\varphi}+\varphi \in n\left\{\left[(\tilde{u}-u)-\alpha_{0}(\tilde{\varphi}-\varphi)\right]+\beta \mathrm{g}(\dot{u})\right\},
\end{array}
$$

where the overhead dot denotes differentiation with respect to the scaled time, i.e., $\dot{x}:=\mathrm{d} x / \mathrm{d} \tau$, and the tilde a delayed quantity, i.e., $\tilde{x}:=x\left(\tau-\tau_{n}\right)$. The set-valued function $g(\dot{u}(\tau))$ models the frictional contact and is defined as

$$
g(\dot{u}(\tau)) \in \frac{\lambda}{2}\left[1-\operatorname{Sign}\left(\dot{u}(\tau)+v_{0}\right)\right]
$$

with the Sign operator given in equation (8). In case the bit sticks axially, i.e., $\dot{u}=-v_{0}$, the magnitude of $g(\dot{u})$ is given by the condition of axial equilibrium of the system,

$$
g\left(\dot{u}=-v_{0}\right)=u-\tilde{u}+\alpha_{0}(\tilde{\varphi}-\varphi) .
$$

The implicit condition for the delay, given in equation (6), has been directly incorporated in the system of equations (15)-(16), noting that the perturbation to the steady-state delay, $\tau_{n}-\tau_{n 0}$, can directly be expressed in terms of the current and delayed perturbations of the bit angular position

$$
\tilde{\varphi}-\varphi=\omega_{0}\left(\tau_{n}-\tau_{n 0}\right)
$$

Thus, if the delay is constant and equal to its steady-state value $\tau_{n 0}$, i.e., the angular velocity of the bit is constant, the angular perturbation is constant, $\varphi=\tilde{\varphi}$, and the term in $\alpha_{0}$ disappears from the evolution equations (15)-(16). Conversely, a constant angular perturbation leads to a constant delay, $\tau_{n}=\tau_{n 0}$.

Equations (15)-(16) are valid as long as the bit is drilling. They lose their physical significance, otherwise. Specifically, the bit/rock interaction laws lose their validity when the bit experiences backward rotation $\left(\dot{\varphi}(\tau)<-\omega_{0}\right)$, 
torsional stick or bouncing. In handling stick-slip oscillations, we assume that the vertical motion sticks as well whenever the bit enters a phase of torsional stick. The vanishing velocities thus correspond to

$$
\dot{\varphi}(\tau)=-\omega_{0}, \quad \dot{u}(\tau)=-v_{0},
$$

as long as the bit sticks. The stick phase ends when the torque built up by the twisting of the drillstring becomes sufficient to overcome the resisting cutting and frictional torques. Bit-bouncing takes place when the axial vibrations experienced by the bit are large enough for the bit to disengage completely from the rock; in which case, the depth of cut becomes negative,

$$
\delta(\tau)=n\left(v_{0} \tau_{n}+(u-\tilde{u})\right)<0,
$$

and the bit is free to move in the borehole, according to the drillstring dynamics.

Modeling stick-slip vibrations or bit-bouncing dynamics requires equations (15) to (19) to be complemented by specific dynamics whenever the system is in stick-slip or bit-bouncing motion. Such extensions are not considered in the stability analysis presented next, as it focuses on the stability of the steady-state response corresponding to drilling at constant velocity.

\section{Linear Stability Analysis of the Coupled Dynamics with State-Dependent Delay}

\subsection{Preamble}

The RGD model takes the form of a system of discontinuous state-dependent delayed differential inclusions. Nonlinearities of two natures are present in the governing equations, namely (i) the discontinuity associated with the function $g(\dot{u}(\tau))$, and (ii) the dependency of the delay on the state of the system. Whereas the former vanishes from the equations upon consideration of trajectories sufficiently close to the steady-state one, an assumption that simultaneously reduces the differential inclusions to differential equations, the second requires specific treatment to ensure that the linearized problem is the variational equivalent of the original nonlinear one and that the stability analysis based on the linear system extends to the nonlinear one.

In this respect, we follow the linearization approach presented in $[16,17]$, which is itself based on the proof proposed in [24]. It relies on the stability assessment by localization in the complex plane of the roots of the characteristic equation associated with an equivalent linear system, i.e., a system with constant delay that has stability properties identical to those of the original system. Starting from the equations stated in the neighborhood of the steady-state (trivial) solution, that is, the governing equations linearized with respect to inclusion $g(\dot{u}(\tau))$,

$$
\begin{aligned}
\ddot{u} & =n \psi\left[(\tilde{u}-u)-\alpha_{0}(\tilde{\varphi}-\varphi)\right], \\
\ddot{\varphi}+\varphi & =n\left[(\tilde{u}-u)-\alpha_{0}(\tilde{\varphi}-\varphi)\right],
\end{aligned}
$$

with the delayed quantities function of the actual delay $\tilde{x}:=x\left(\tau-\tau_{n}\right)$, linearization with respect to the state-dependent delay can be performed under the cover of three different assumptions. Nevertheless, only one proves to be equivalent 
to the original one when it comes to assessing the stability from numerically computed time responses, as will be shown.

Timescale separation: Assuming $\psi \gg 1$, the axial and torsional dynamics can be shown to evolve on different timescales. Indeed, under that hypothesis, the equation ruling the axial dynamics takes the form of a singularly perturbed problem, and it ensues that the evolution of the axial dynamics takes place on a faster timescale than that of the angular motion. Accordingly, the axial motion can be studied independently of the torsional one for the latter appears constant on the fast timescale. The timescale separation is thus equivalent to a decoupling of the system, which now has a constant delay on the fast timescale, and the study of the response stability amounts to the stability analysis of two uncoupled oscillators $[2,19]$.

The torsional pendulum

$$
\ddot{\varphi}+\varphi=0
$$

is marginally stable while the axial motion, governed by equation

$$
\ddot{u}=n \psi(\breve{u}-u)
$$

with $\breve{u}:=u\left(\tau-\tau_{n 0}\right)$, is asymptotically stable provided the steady-state delay (rotation speed $\omega_{0}$ ) is smaller (larger) than the critical value $\tau_{c}^{0}=\pi / \sqrt{2 n \psi}\left(\omega_{c}^{0}=\sqrt{8 \psi / n}\right)$. Details of the analysis can be found in [2], while some of its shortcomings are demonstrated in [19] where trajectories of the system are shown to slowly diverge from the trivial solution for configurations of stable axial motion.

Constant delay: According to equation (19), the assumption $\tau_{n}=\tau_{n 0}, \forall \tau>0$ implies a constant angular velocity of the bit and $\varphi=\tilde{\varphi}$. The equivalent problem then reads

$$
\begin{aligned}
\ddot{u} & =n \psi(\breve{u}-u), \\
\ddot{\varphi}+\varphi & =n(\breve{u}-u),
\end{aligned}
$$

and it appears that the assumption of constant delay leads to a partial decoupling of the axial and torsional dynamics. They are now serially connected, with the independent axial motion driving the torsional one. This formulation neglects the dynamics of the state-dependent delay. It is analyzed in Section 3.3.

State-dependent delay: For the linearized equations to hold as the variational equivalent of the original state-dependent delayed differential equations (SD-DDE), the dynamics of the delay must be accounted for during linearization. Starting from the generic first-order representation of an autonomous system of SD-DDE,

$$
\dot{\mathbf{x}}(t)=\mathbf{f}\left(\mathbf{x}(t), \mathbf{x}\left(t-\tau\left(\mathbf{x}_{t}\right)\right), \tau\left(\mathbf{x}_{t}\right)\right)
$$

with state vector $\mathbf{x} \in \mathbb{R}^{m}$, the variational equivalent around the constant solution $\overline{\mathbf{x}}$ is given by Insperger $e t$ al. $[16,17]$ as

$$
\dot{\mathbf{y}}(t)=\mathrm{D}_{1} \mathbf{f}\left(\overline{\mathbf{x}}, \overline{\mathbf{x}}, \tau\left(\overline{\mathbf{x}}_{t}\right)\right) \mathbf{y}(t)+\mathrm{D}_{2} \mathbf{f}\left(\overline{\mathbf{x}}, \overline{\mathbf{x}}, \tau\left(\overline{\mathbf{x}}_{t}\right)\right) \mathbf{y}\left(t-\tau\left(\overline{\mathbf{x}}_{t}\right)\right)+\mathrm{D}_{3} \mathbf{f}\left(\overline{\mathbf{x}}, \overline{\mathbf{x}}, \tau\left(\overline{\mathbf{x}}_{t}\right)\right) D \tau\left(\overline{\mathbf{x}}_{t}\right) \mathbf{y}_{t}
$$


where $\mathbf{y} \in \mathbb{R}^{m}$ denotes the vector of perturbations around the constant solution, and $\mathrm{D}_{i} \mathbf{f}, i=1,2,3$ refer to the derivative of the vector field $\mathbf{f}$ with respect to its $i^{\text {th }}$ argument. Operator $\mathrm{D} \tau$ must be understood as the Frchet derivative of the time-delay with respect to the $\mathbf{x}_{t}$. Thus, the linearized problem has a constant delay but accounts for the delay dynamics through the last term of equation (27). Application of the linearization procedure to equations (22) comes down to evaluating them at the steady-state delay

$$
\begin{aligned}
\ddot{u} & =n \psi\left[(\breve{u}-u)-\alpha_{0}(\breve{\varphi}-\varphi)\right], \\
\ddot{\varphi}+\varphi & =n\left[(\breve{u}-u)-\alpha_{0}(\breve{\varphi}-\varphi)\right],
\end{aligned}
$$

for the dynamics of the delay is already stated in terms of the state variables in the governing equations. This linearized formulation is in line with the approach proposed in [24] and will appear as the correct one for the assessment of the steady-state response stability.

As a notation convention, for the remainder of this section, we denote by subscript ${ }_{0}$ all quantities that refer to the steady-state; the superscript ${ }^{0}$ is used for reference to the formulation with constant delay.

Further details on the stability analysis of state-dependent delayed differential equations and their applications can be found, among others, in [16, 18, 25-29] and the references therein.

\subsection{Characteristic Equation}

Similarly to the stability analysis of the equilibria of a linear ordinary differential equation, the stability of the stationary response of a linear delayed differential equation can be studied by considering the location of its associated poles in the complex plane; that is, the zeros of its characteristic equation resulting from the typical exponential Ansatz. Any pole having a positive real part will then be responsible for the instability of the equilibrium whereas asymptotic stability will be concluded if all poles have a negative real part.

Following the developments of Appendix A, the characteristic equation based on the formulation including the delay dynamics, i.e., the system of equations (28), is given by the following exponential-polynomial

$$
P(s):=\left(s^{2}+1\right)\left(s^{2}+n \psi\left(1-\mathrm{e}^{-s \tau_{n 0}}\right)\right)-n \alpha_{0} s^{2}\left(1-\mathrm{e}^{-s \tau_{n 0}}\right)=0 .
$$

Exponential-polynomials of the above type admit infinitely many roots [30, 31]. Nonetheless, only a finite number of them have a positive real part. Investigating the stability of the system can then be performed by tracking the crossings of the imaginary axis by the system poles as a parameter is varied, the steady-state delay $\tau_{n 0}$ or nominal rotation speed $\omega_{0}=2 \pi / n \tau_{n 0}$ in the present case. Calculations show that there is always at least one pair of poles with positive real part for $\tau_{n 0}>0$. Accordingly, steady drilling is never stable as already noted in [14, 32]. However, two regimes of instability can be distinguished: a "slow" one and a "fast" one. We respectively associate them with a dominant torsional or axial instability, by reference to the torsional and axial poles of the uncoupled system. The boundary between these two regimes can be expressed in terms of a critical delay $\tau_{n c}=\tau_{n c}\left(n \psi, n \alpha_{0}\right)$, with $\tau_{n 0}<\tau_{n c}$ corresponding to the slow regime, and $\tau_{n 0}>\tau_{n c}$ to the fast one. 
Before studying the influence of the delay $\tau_{n 0}$ and of the parameters $\left(n \psi, n \alpha_{0}\right)$ on the location in the complex plane of the characteristic roots of $P(s)=0$, it is useful to note that $P(s)$ can be expressed as

$$
P(s)=P^{0}(s)-n \alpha_{0} \Delta P(s)
$$

where

$$
P^{0}(s) \equiv\left(s^{2}+1\right)\left(s^{2}+n \psi\left(1-\mathrm{e}^{-s \tau_{n o}}\right)\right)
$$

and

$$
\Delta P(s) \equiv s^{2}\left(1-\mathrm{e}^{-s \tau_{n 0}}\right)
$$

This particular decomposition of $P(s)$ enables the study of the stability of the coupled axial/torsional dynamics with state-dependent delay by reference to the dynamics with the delay assumed to be constant, cf. (25), whose stability is associated with the characteristic equation $P^{0}(s)=0$.

\subsection{Stability of the Axial/Torsional Dynamics with Constant Delay}

The exponential-polynomial $P^{0}(s)$ consists of the product of two functions: the characteristic polynomial $\left(s^{2}+1\right)$ relative to the undamped torsional pendulum $\ddot{\varphi}+\varphi=0$ and the characteristic function $P_{\mathrm{a}}(s):=s^{2}+n \psi\left(1-\mathrm{e}^{-s \tau_{n o}}\right)$ associated with the axial dynamics. The latter is indeed recovered from equation (24), under the assumption of timescale separation. Thus, under the assumption of constant delay, a decoupling of the axial and torsional degrees of freedom takes place in the assessment of the steady-state response stability, as was already noted from the serial connection observed in equations (25).

Associated with the torsional dynamics are two imaginary poles at $s_{t}= \pm \mathrm{j}$, whereas the axial dynamics has infinitely many. Their location depends on the delay $\tau_{n 0}$ and parameter $n \psi$, and is limited to some left part of the complex plane. The exponential-polynomial $P_{a}(s)$ belongs to the general class studied in $[30,31,33]$ and closely relates to those arising in the study of tool chatter in machining processes [34, 35]. Its stability analysis can be found in $[2,13]$. These studies show that $P_{a}(s)=0$ has a root at the origin of the complex plane, regardless of the positive values $n \psi$ and $\tau_{n 0}$ take, and that this pole is to be related to the fact that the solution to equation (24) is undefined with respect to a position offset. As such, it can be discarded in the assessment of the steady-state stability. They also prove that $P_{a}(s)=0$ admits one pair of imaginary conjugate poles $s_{c}= \pm \mathrm{j} \varpi_{*}^{0}$, at discrete values $\tau_{m}^{0}$ of the delay $\tau_{n 0}$ given by

$$
\tau_{m}^{0}=\frac{(2 m-1) \pi}{\varpi_{*}^{0}}, \quad m \in \mathbb{Z}^{+}:=\{1,2, \cdots\},
$$

with $\varpi_{*}^{0}=\sqrt{2 n \psi}$. Treating the delay $\tau_{n 0}$ as a bifurcation parameter, it can readily be shown that the axial system is stable if and only if the delay is smaller than the critical value

$$
\tau_{n c}^{0}=\tau_{1}^{0}=\frac{\pi}{\sqrt{2 n \psi}},
$$

at which the rightmost pair of conjugated roots of $P_{a}(s)$ (besides the pole at the origin) crosses the imaginary axis to enter the half-plane $\operatorname{Re}(s)>0$. In other words, all the roots of $P^{0}(s)=0$ but the two torsional poles $s_{t}$ and the one 
at the origin are located in the half-plane $\operatorname{Re}(s)<0$, if $\tau_{n 0}<\tau_{n c}^{0}$. Accordingly, the formulation assuming a coupled system with constant delay predicts that steady drilling is either unstable or marginally stable depending on whether the delay $\tau_{n 0}$ is larger or smaller than the critical delay $\tau_{n c}^{0}$. The instability is associated with the axial dynamics poles, which we refer to as axial instability.

Since the freezing of the delay uncouples the axial and torsional motions, the criterion for instability matches the one predicted under the hypothesis of timescale separation between the axial and torsional dynamics.

\subsection{Stability of the Axial/Torsional Dynamics with State-Dependent Delay}

To analyze the stability of system (28), we track the location in the complex plane of the most unstable poles of its characteristic equation (30), i.e., the ones with the largest positive real part, as the steady-state delay is varied. As usual, the analysis starts by computing the conditions for which there are critical eigenvalues of the form $s_{c}= \pm \mathrm{j} \varpi$. These occur for specific values of the steady-state delay.

Searching for imaginary roots to $P(s)=0$ leads to the following system of equations to be solved for the pulsation $\varpi>0$ and for the delay $\tau_{n 0}>0$

$$
\begin{aligned}
\left(1-\varpi^{2}\right)\left(n \psi\left(1-\cos \varpi \tau_{n 0}\right)-\varpi^{2}\right)+n \alpha_{0} \varpi^{2}\left(1-\cos \varpi \tau_{n 0}\right) & =0, \\
\left(n \psi\left(1-\varpi^{2}\right)+n \alpha_{0} \varpi^{2}\right) \sin \varpi \tau_{n 0} & =0 .
\end{aligned}
$$

Besides the existence of a pole at the origin of the complex plane, again related to the invariance of the steady-state response to a displacement offset, there are three pairs of possible crossing points. The first one corresponds to the location of the torsional poles under a decoupling assumption

$$
s_{c}= \pm \mathrm{j}
$$

and occurs at critical delays

$$
\tau_{2 m}=2 m \pi, \quad m \in \mathbb{Z}^{+} .
$$

The two other crossings take place at $s_{c}= \pm \mathrm{j} \varpi_{*}^{+}$and $s_{c}= \pm \mathrm{j} \varpi_{*}^{-}$, with pulsations and critical delays given by

$$
\begin{aligned}
\varpi_{*}^{ \pm} & =\sqrt{1 / 2+n\left(\psi-\alpha_{0}\right) \pm \sqrt{n^{2}\left(\psi-\alpha_{0}\right)^{2}-n\left(\alpha_{0}+\psi\right)+1 / 4}}, \\
\tau_{m}^{ \pm} & =\frac{(2 m-1) \pi}{\varpi_{*}^{ \pm}}, \quad m \in \mathbb{Z}^{+},
\end{aligned}
$$

where the superscript ${ }^{ \pm}$sign is associated with the \pm sign in the definition of the pulsation $\varpi_{*}^{ \pm}$. The existence of the crossings at $s_{c}= \pm \mathrm{j} \varpi_{*}^{ \pm}$is contingent, however, on the positiveness of the square root arguments in (38). Two conditions thus exist: $C_{1}:=n^{2}\left(\psi-\alpha_{0}\right)^{2}-n\left(\alpha_{0}+\psi\right)+1 / 4>0$ and $C_{2}:=1 / 2+n\left(\psi-\alpha_{0}\right)-\sqrt{C_{1}}>0$. It can readily be shown that $C_{1}$ is indeed positive if $0<\alpha_{0}<\alpha_{0}^{-}$or $\alpha_{0}>\alpha_{0}^{+}$, with $n \alpha_{0}^{ \pm}=1 / 2+n \psi \pm \sqrt{2 n \psi}$. Furthermore, we also have $C_{2}>0$ under the restriction that $0<\alpha_{0}<\alpha_{0}^{-}$.

Figure 3 illustrates the division of the parametric space $\left(n \psi, n \alpha_{0}\right)$ according to the signs of $C_{1}$ and $C_{2}$; the dashed line denotes the oblique asymptote to which $n \alpha_{0}^{ \pm}$converge when $n \psi \gg 1$. It is divided in three regions I, II, III that 


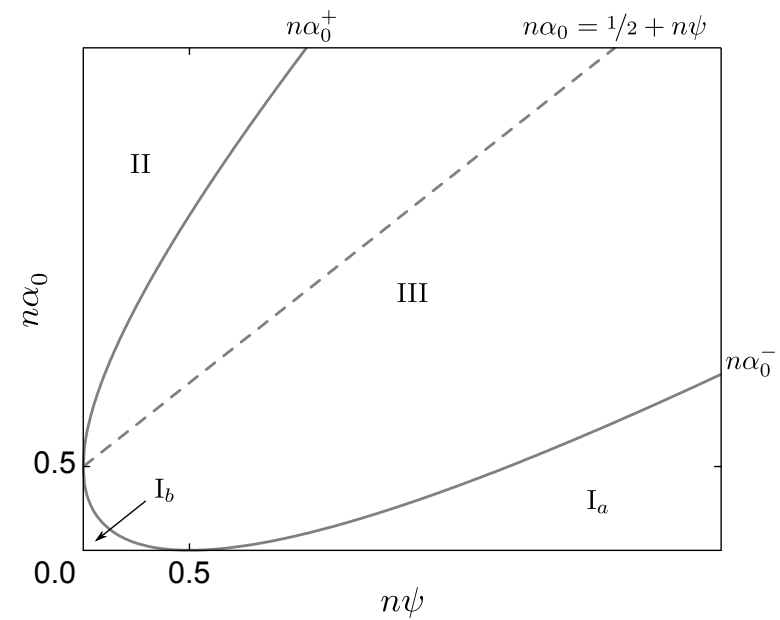

Figure 3: The number of crossing points of the eigenvalue root locus on the imaginary axis of the complex plane depends on the value of parameters $\left(n \psi, n \alpha_{0}\right)$. Region I corresponds to 3 crossing points at $s_{c}= \pm \mathrm{j}, s_{c}= \pm \mathrm{j} \varpi_{*}^{+}$and $s_{c}= \pm \mathrm{j} \varpi_{*}^{-}$. The sole crossing point $s_{c}= \pm \mathrm{j}$ is to be found in regions II and III.

are separated by the loci $\alpha_{0}=\alpha_{0}^{ \pm}$corresponding to $C_{1}=0$. These regions are associated with different qualitative behaviors of the root locus of the characteristic equation, with three pairs of crossing points for region $\mathrm{I}\left(0<\alpha_{0}<\alpha_{0}^{-}\right)$, but only crossings at $s_{c}= \pm \mathrm{j}$ in regions II and III $\left(\alpha_{0}>\alpha_{0}^{-}\right)$. Region I further subdivides into $\mathrm{I}_{a}$ and $\mathrm{I}_{b}$ at $n \psi=1 / 2$, which corresponds to $\alpha_{0}^{-}=0$.

Whatever the parametric configuration or region, the analysis of the eigenvalue rootlocus shows that the system is always unstable, due to the permanent presence of poles in the right-hand side (RHS) of the complex plane for a positive delay $\tau_{n 0}>0$; see next section and Appendix B. Moreover, it appears that, for a vanishing delay $\left(\tau_{n 0}=0\right)$, there are always two poles located at $s_{c}=s_{t}= \pm \mathrm{j}$ and that these poles move into the RHS of the complex plane as the delay is increased from zero. By reference to the analysis of the uncoupled equations, we define these two poles as the torsional or angular poles and the infinitely many remaining ones as the axial poles. We then relate the type of instability to the set to which the dominant unstable poles belong, as explained in the next section that refines the stability analysis in region $\mathrm{I}_{a}$.

\section{Modes of Instability in Deep Drilling Systems}

Deep drilling systems are typically characterized by $\alpha_{0}=O\left(10^{-1}\right), \psi=O\left(10-10^{3}\right)$, and $n \geq 4$. Thus, clearly only region $\mathrm{I}_{a}\left(0<\alpha_{0}<\alpha_{0}^{-}, n \psi>1 / 2\right)$ of the parameter space $\left(n \psi, n \alpha_{0}\right)$ is relevant for these systems. Other regions might be of interest for other applications, such as metal turning, in which case the system parameter $\psi$ would be much smaller given the stiffer nature of the tool [17]. The reader is referred to Appendix D for the qualitative description of the root locus of the system poles in the complex plane, when the parameters $\left(n \psi, n \alpha_{0}\right)$ are outside region $\mathrm{I}_{a}$. 


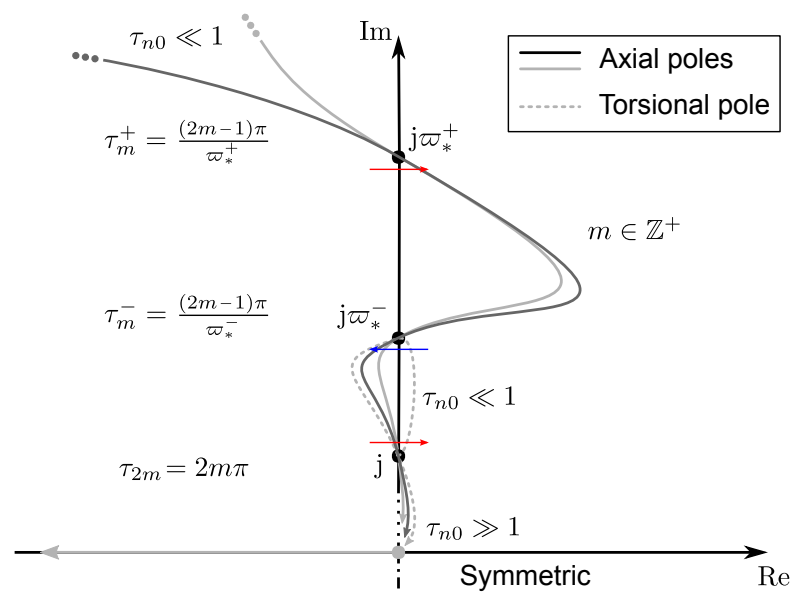

Figure 4: Qualitative sketch of the pole trajectories upon variation of delay $\tau_{n 0}$, for parameters $\left(n \psi, n \alpha_{0}\right)$ belonging to region $\mathrm{I}_{a}$ (see Figure 3 ). As the delay is increased from 0 , the torsional poles leave $s_{c}= \pm \mathrm{j}$ towards $s_{c}= \pm \mathrm{j} \varpi_{*}^{-}$. Simultaneously, one pole moves from the origin toward the LHS of the complex plane while the axial poles approach $s_{c}= \pm \mathrm{j} \varpi_{*}^{+}$from the LHS. Further increasing the delay leads to crossings from LHS to RHS at $s_{c}= \pm \mathrm{j} \varpi_{*}^{+}$and $s_{c}= \pm \mathrm{j}$ for delays $\tau_{n 0}=\tau_{m}^{+}$and $\tau_{n 0}=\tau_{2 m}$ with $m=1,2, \cdots$, respectively. Crossings from RHS to LHS take place at $s_{c}= \pm \mathrm{j} \varpi_{*}^{-}$for $\tau_{n 0}=\tau_{m}^{-}, m=1,2, \cdots$; the first one corresponds to the return of the torsional poles in the LHS. Given the inequality $\tau_{1}^{+}<\tau_{1}^{-}$, the system instability is dominated by the axial poles once $\tau_{n 0}>\tau_{n c} \simeq \tau_{1}^{+}$, whereas it is the torsional dynamics that rules the unstable behavior for $\tau_{n 0}<\tau_{n c}$. These define two instability regimes: a fast one associated with the axial dynamics (unstable poles with a large real part) and a slow one related to the torsional dynamics (unstable poles with a small real part).

\subsection{Influence of Rotation Speed}

If $0<\alpha_{0}<\alpha_{0}^{-}$, the roots of the characteristic equation (29), $P(s)=0$, cross the imaginary axis at three conjugated locations, namely, $s_{c}= \pm \mathrm{j}, s_{c}= \pm \mathrm{j} \varpi_{*}^{+}$, and $s_{c}= \pm \mathrm{j} \varpi_{*}^{-}$. In addition, for parameters belonging to region $\mathrm{I}_{a}$, we can show that $\varpi_{*}^{+}>\varpi_{*}^{-}>1$ and the crossings at $s_{c}= \pm \mathrm{j}$ and at $s_{c}= \pm \mathrm{j} \varpi_{*}^{+}$are from the left-hand side (LHS) to the right-hand side (RHS) of the complex plane, whereas those at $s_{c}= \pm \mathrm{j} \varpi_{*}^{-}$are from RHS to LHS. The calculation of the crossing directions is given in Appendix B.

We are now in a position to study the motion of the eigenvalues of the linearized system in the complex plane as the delay $\tau_{n 0}$, or equivalently the angular velocity $\omega_{0}$, changes. Figure 4 provides a qualitative representation of the eigenvalue trajectories as the delay $\tau_{n 0}$ is increased from 0 to positive values. They move as follows.

1. At the limit $\tau_{n 0}=0$ (corresponding to an infinitely fast rotation speed of the bit), there is a double pole at the origin and a pair of conjugated poles located at $s_{t}= \pm \mathrm{j}$. One of the poles at the origin is to be discarded in the stability analysis.

2. With $\tau_{n 0}$ increasing from 0 , the poles associated with the torsional dynamics, originally at $s_{t}= \pm \mathrm{j}$, move slightly to the right of the imaginary axis, i.e., $\operatorname{Re}\left(s_{t}\right)=\epsilon$, with $0<\epsilon\left(\tau_{n 0}, n \psi, n \alpha_{0}\right) \ll 1$. One of the poles at the origin enters the LHS of the complex plane.

3. At $\tau_{n 0}=\tau_{1}^{+}:=\pi / \varpi_{*}^{+}$, a first conjugated pair of axial poles crosses the imaginary axis at $s_{c}= \pm \mathrm{j} \varpi_{*}^{+}$. For all practical purposes, this pair of axial poles can be treated as the most unstable poles, i.e., they can be considered to be on the right of the torsional poles, once $\tau_{n 0}>\tau_{1}^{+}$. 
4. At $\tau_{n 0}=\tau_{1}^{-}:=\pi / \varpi_{*}^{-}\left(>\tau_{1}^{+}\right)$, the torsional poles, which have left $s_{t}= \pm \mathrm{j}$ when $\tau_{n 0}$ increased from 0 , return to the half-plane $\operatorname{Re}(s)<0$ by crossing the imaginary axis at $s_{c}= \pm \mathrm{j} \varpi_{*}^{-}$. The pair will reside in $\operatorname{Re}(s)<0$ until $\tau_{n 0}=\tau_{2}:=2 \pi$ where it returns to the half-plane $\operatorname{Re}(s)>0$ at $s_{c}= \pm \mathrm{j}$.

5. At $\tau_{n 0}=\tau_{3}^{+}$, a second pair of axial poles crosses the imaginary axis at $s_{c}= \pm \mathrm{j} \varpi_{*}^{+}$, moving from left to right.

6. As $\tau_{n 0}$ increases from $\tau_{1}^{+}$to $\tau_{3}^{-}$, the first pair of axial poles moves further to the right of $\operatorname{Re}(s)=0$ to eventually turn around and move towards the imaginary axis, which they cross at $s_{c}= \pm \mathrm{j} \varpi_{*}^{-}$from right to left at $\tau_{n 0}=$ $\tau_{3}^{-}:=3 \pi / \varpi_{*}^{-}$. The second pair of axial poles follows a similar trajectory as $\tau_{n 0}$ increases from $\tau_{3}^{+}$to $\tau_{5}^{-}$.

7. The pairs of axial poles (in infinite number) located in $\operatorname{Re}(s)<0$ move to the right, with sequential crossings of the imaginary axis taking place at $\tau_{n 0}=\tau_{5}^{+}, \tau_{7}^{+}, \cdots$; these pairs follow a trajectory similar to that of the first pair, as they eventually cross again the imaginary axis from right to left at $s_{c}= \pm \mathrm{j} \varpi_{*}^{-}$at delays corresponding to $\tau_{n 0}=\tau_{7}^{-}, \tau_{9}^{-}, \cdots$ and return to the half-plane $\operatorname{Re}(s)>0$ at $s_{c}= \pm \mathrm{j}$ at delays $\tau_{n 0}=\tau_{4}, \tau_{6}, \cdots$.

A systematic parametric exploration of the influence of $n \psi$ and $n \alpha_{0}$ on the solution confirms the above narrative on the dependence of the two sets of characteristic roots on the delay $\tau_{n 0}$.

In particular, in view of the fact that $\varpi_{*}^{+}>\varpi_{*}^{-}>1$ in region $\mathrm{I}_{a}$, there will always be axial dynamics poles in the RHS for $\tau_{n 0}>\tau_{1}^{+}$, with $\tau_{1}^{+}$(the delay at which the first pair of axial poles crosses the imaginary axis) given by

$$
\tau_{1}^{+}=\frac{\pi}{\sqrt{1 / 2+n\left(\psi-\alpha_{0}\right)+\sqrt{n^{2}\left(\psi-\alpha_{0}\right)^{2}-n\left(\alpha_{0}+\psi\right)+1 / 4}}} .
$$

Moreover, for $\tau_{n 0}>\tau_{n c}$, with $\tau_{n c}$ denoting the critical delay at which the angular and rightmost axial poles have the same (positive) real part, the axial poles are always the most unstable ones. Finally, given the proximity of the angular poles to the imaginary axis, $\tau_{n c}$ can be approximated by the delay $\tau_{1}^{+}$corresponding to the first crossing of the imaginary axis by axial poles. Appendix $\mathrm{C}$ provides an approximate expression for the real part of the most unstable slow pole when $\tau_{n 0}<\tau_{1}^{+}$; that is, when torsional poles are responsible for the instability of the steady-state.

These important results can be summarized in terms of the angular velocity $\omega_{0}$. There is a critical velocity $\omega_{c}$, which marks the transition between two regimes of instability: unstable axial dynamics $\left(\omega_{0}<\omega_{c}\right)$ and unstable torsional dynamics $\left(\omega_{0}>\omega_{c}\right)$. This transition rotation speed can be approximated by $\omega_{1}^{+}=2 \pi / n \tau_{1}^{+}$, i.e.,

$$
\omega_{c} \simeq \frac{2}{n} \sqrt{1 / 2+n\left(\psi-\alpha_{0}\right)+\sqrt{n^{2}\left(\psi-\alpha_{0}\right)^{2}-n\left(\alpha_{0}+\psi\right)+1 / 4}}
$$

which can be further simplified to

$$
\omega_{c} \simeq \omega_{c}^{0}=\sqrt{\frac{8 \psi}{n}}
$$

since $\alpha_{0} \ll \psi$ in deep drilling applications. In other words, the transition rotation speed $\omega_{c}$ corresponds for all practical purposes to $\omega_{c}^{0}$, the angular velocity at which the axial dynamics becomes unstable when the delay remains constant. 

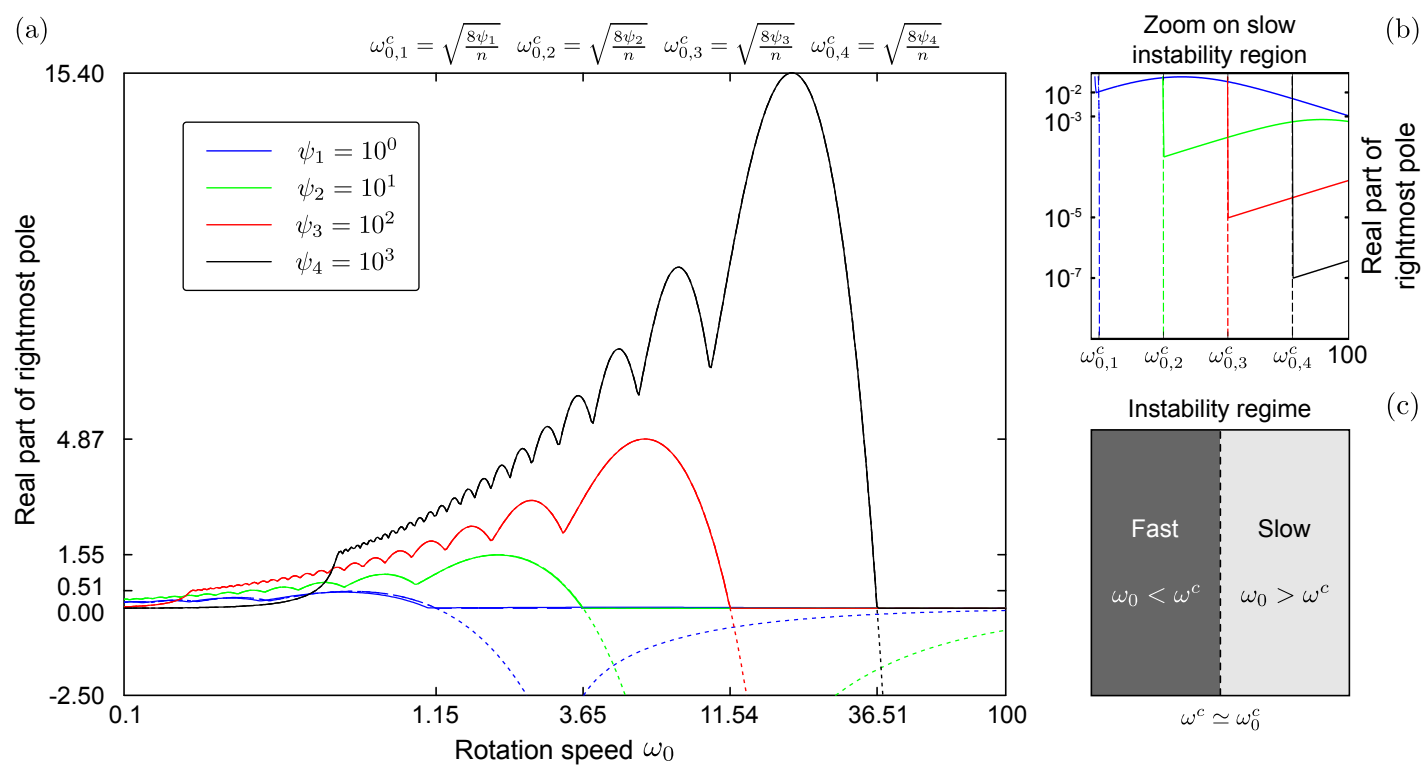

b)

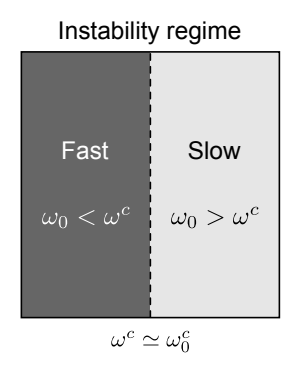

(c)

Figure 5: Influence of parameter $\psi$ on the rightmost pole of the system, $\left(n, \alpha_{0}\right)=(6,0.1)$. Insets (a) and (b): Comparison of the three linearized forms given by equations (24) to (28): axial dynamics (dotted line), constant delay (dashed line) and state-dependent delay (solid line). While the analysis based on the sole axial dynamics predicts stability for rotation speeds above the critical velocity (negative real part of dominant pole), the formulation based on the constant delay predicts marginal stability (zero real part of dominant pole), whereas the formulation with the statedependent delay predicts instability (positive real part of dominant pole); the latter result is the one observed in numerical simulations of the system behaviors by direct time integration. Insets (b) and (c): Two instability regimes can then be identified based on the magnitude of the real part of the dominant unstable pole: a "slow" and a "fast" unstable regimes. The transition from one regime to the other takes place at a critical rotation speed that is well approximated by equation (41).

\subsection{Fast and Slow Regimes of Instability}

The above stability analysis has demonstrated the influence of the nominal rotation speed on the positions of the system poles in the complex plane and the existence of a critical rotation speed corresponding to a change of influence on the system response between the torsional and axial poles. Moreover, it has been shown that, for the range of parameters relevant to deep drilling, the torsional poles remain in the close neighborhood of the imaginary axis upon variation of the rotation speed, while the axial poles exhibit larger excursions from this axis. The dominance of unstable poles of one category or the other therefore defines two types of system behavior in response to external perturbations. Namely, the system will exhibit slow divergence if the angular poles dominate and fast divergence if the axial ones do, as the rapidity and strength with which the system reacts to an external perturbation is directly related to the magnitude of the real part of its dominant poles-similarly to non-delayed systems. We refer to these two categories as slow and fast instabilities and consider the associated configuration as a slow or a fast one.

The profound difference between the two modes of instability, as well as the sharp transition between the two regimes that is taking place at $\omega_{0}=\omega_{c} \simeq \omega_{c}^{0} \equiv \sqrt{8 \psi / n}$ is well illustrated by Figure 5. For four values of the system parameter $\psi$, it depicts the variation of the real part of the system rightmost pole with the nominal speed $\omega_{0}=2 \pi / n \tau_{n 0}$; that is, the dominant unstable pole. The curves obtained when considering the sole axial dynamics or neglecting the delay dynamics in assessing the steady-state stability have also been included for comparison. For rotation speeds 
above the critical velocity, the rightmost pole has a negative real part when considering the axial dynamics only (dotted lines), a stable steady-state is thus predicted, while the formulation with constant delay predicts marginal stability due to the presence of the torsional poles at $s_{t}= \pm \mathrm{j}$ (dashed lines), i.e., it is inconclusive. The formulation accounting for the delay dynamics predicts the instability of the steady-state response due to the presence of the torsional poles in the RHS (solid lines). The real part of the torsional poles remains, however, very limited; see inset (b) of Figure 5. The latter result is numerically demonstrated to be correct in the next section.
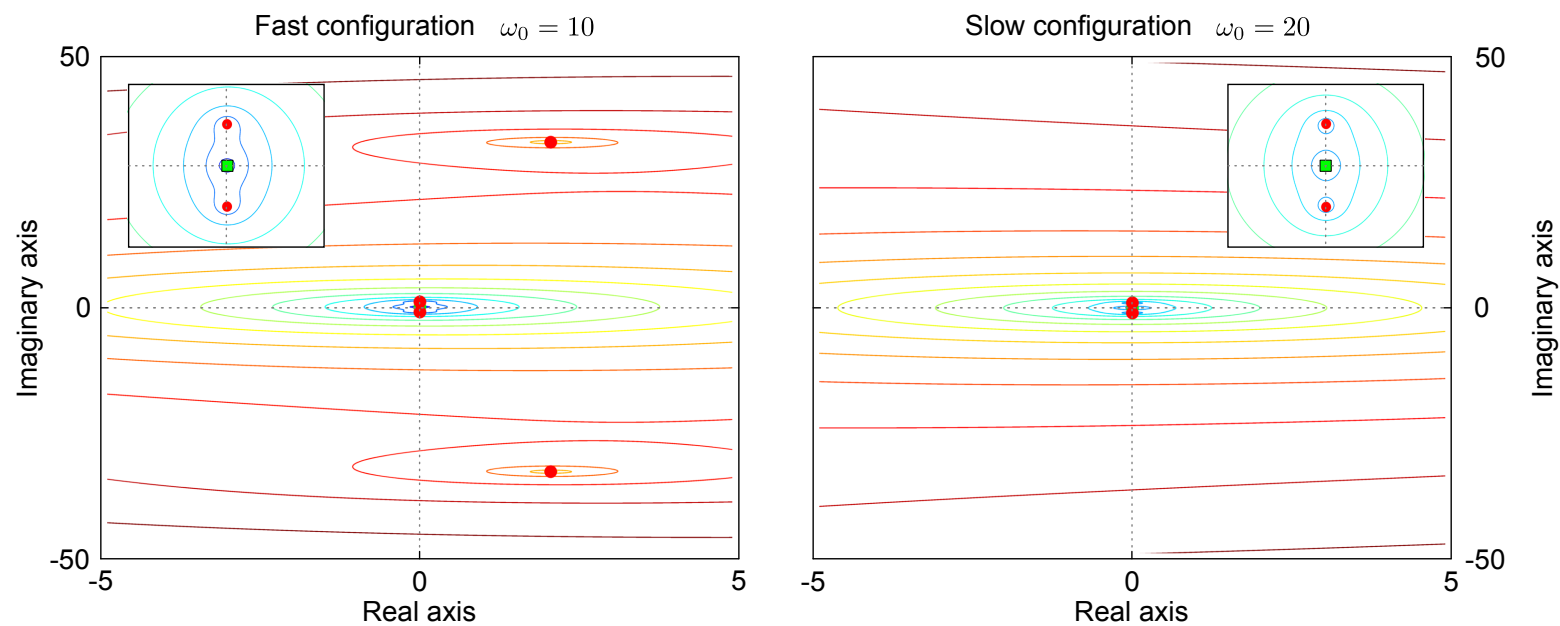

Figure 6: Locations of the system poles in the complex plane for a fast configuration $\left(\omega_{0}=10\right.$, left $)$ and a slow one $\left(\omega_{0}=20\right.$, right $)$, with $\left(n, \psi, \alpha_{0}\right)=(6,100,0.1)$. The fast configuration is dominated by two axial poles with real part 2.05 while the slow one is lightly unstable due to two torsional poles with real part $1.6 \cdot 10^{-5}$. Red disks denote unstable poles while green squares correspond to marginally stable ones. Level-set curves reflect the magnitude of the characteristic function $|P(s)|$. The roots of the characteristic equation have been computed using Matlab toolbox DDE-BIFTOOL [36]. The respective insets represent the neighborhood of the origin of the complex plane.

This difference in behavior is further illustrated in Figure 6 that shows the poles of the linearized system (28) for both a fast and a slow parametric configurations. For the particular set of parameters used, $\left(n, \psi, \alpha_{0}\right)=(6,100,0.1)$, the approximation of the transition speed is given by $\omega_{c} \simeq 11.5$. Accordingly, $\omega_{0}=10$ and $\omega_{0}=20$ correspond to a fast and a slow configurations respectively, as can be seen from the magnitude of the dominant poles real part, 2.05 for the former case and $1.6 \cdot 10^{-5}$ for the latter one. In the presence of external perturbations, the fast instability leads to the rapid apparition of self-excited oscillations that can degenerate into stick-slip, bit-bouncing or the backward rotation of the bit, contrary to the regime of slow instability where the amplification of external perturbations is a lengthy process on the timescale of bit motion.

\section{Self-Excited Vibrations in Deep Drilling Systems: Coexistence of Drilling Regimes}

In actual drilling conditions, the assumption of homogeneous rock does not hold as the drill bit could experience changing mechanical properties while it penetrates the rock medium; that is, parameters $\varepsilon$ and $\sigma$ vary continuously with the bit depth, resulting in a change of the torque and forces acting on the bit.

A convenient way to assess the influence of perturbations on the system steady-state response is to consider an 
exogenous torque perturbation. In the following, we show that such a perturbation can lead to a change of drilling regime, i.e., an apparently stable drilling configuration could rapidly become the subject of stick-slip vibrations. We consider the slow parametric configuration of Figure 6 , namely $\left(\omega_{0}, n, \psi, \alpha_{0}\right)=(20,6,100,0.1)$, and the application to the bit of an exogenous perturbation under the form of a drag torque of constant magnitude and finite duration; that is, the equations governing the evolution of the perturbations to the steady-state response become

$$
\ddot{u}=n \psi\left(\tilde{u}-u+\alpha_{0}(\varphi-\tilde{\varphi})\right), \quad \ddot{\varphi}+\varphi=n\left(\tilde{u}-u+\alpha_{0}(\varphi-\tilde{\varphi})\right)-\mathcal{T}_{\mathrm{p}}(\tau),
$$

with

$$
\mathcal{T}_{\mathrm{p}}(\tau)= \begin{cases}\mathcal{T}_{\mathrm{p}}^{0} & \text { if } \tau \in\left[\tau_{\text {in }}, \tau_{\text {out }}\right] \\ 0 & \text { otherwise. }\end{cases}
$$

The state-dependent delay is computed according to equation (19). Time integration is carried out on the basis of the algorithm described in Appendix $\mathrm{C}$ of [2]. Two perturbations of equal duration $\tau_{\text {out }}-\tau_{\text {in }}=7.5$ are considered; one with large amplitude and the other one with a moderate amplitude, $\mathcal{T}_{\mathrm{p}}^{0}=0.9 \omega_{0}$ and $\mathcal{T}_{\mathrm{p}}^{0}=0.25 \omega_{0}$ respectively. The resulting responses are shown in Figure 7. In case no external torque is applied to the system, perturbations remain zero (gray), contrary to what is observed for fast configurations where numerical errors are sufficient to trigger the instability [37]. Application of a large external torque rapidly leads to the apparition of stick-slip vibrations $\left(\dot{\varphi}=-\omega_{0}\right)$ after its application (red), with a periodic motion (limit cycle) taking place after the first sticking phase. The second perturbed case leads to a quasiperiodic solution (blue), in the sense that motion is close to periodic on the timescale of bit motion - taken to correspond to the resonance period of the drillstring viewed as a torsional pendulum—but transient on a slower timescale; the trajectory ultimately converges to the stick-slip limit cycle observed in the case of the large perturbation (up to a constant displacement, as the system is invariant under an offset of displacement $u$ ). This observation concurs with that of Germay et al. [12], who have concluded, using a finite element model for the drillstring, that an increased rotation speed at the rig delays the emergence of stick-slip oscillations. This increase, in fact, corresponds to bringing the drilling system in a regime of slow instability. It also meets the conclusion of the experimental investigations of Ledgerwood et al. [38] who showed that increasing the surface rotation speed of the drillstring at constant weight on bit reduces the occurrence of stick-slip oscillations; they note, however, that a too large increase can be responsible for the appearance of backward whirl, a drilling dysfunction during which the bit instantaneous center of rotation rotates backwards around the hole axis.

Accordingly, the model predicts that, on the timescale of the bit motion, different types of motion can be observed for an unstable but slow configuration: (i) perturbations are close to zero, the steady-state response is observed; (ii) perturbations are moderate, the bit exhibits limited-amplitude self-excited oscillations around its stationary trajectory; and (iii) large perturbations can trigger instabilities such as stick-slip oscillations, backward rotation or bit-bouncing. The apparent coexistence of drilling regimes, observed in both laboratory experiments and field, is thus a consequence of the drilling system slow dynamics inherent to a specific parametric configuration or drilling conditions. 


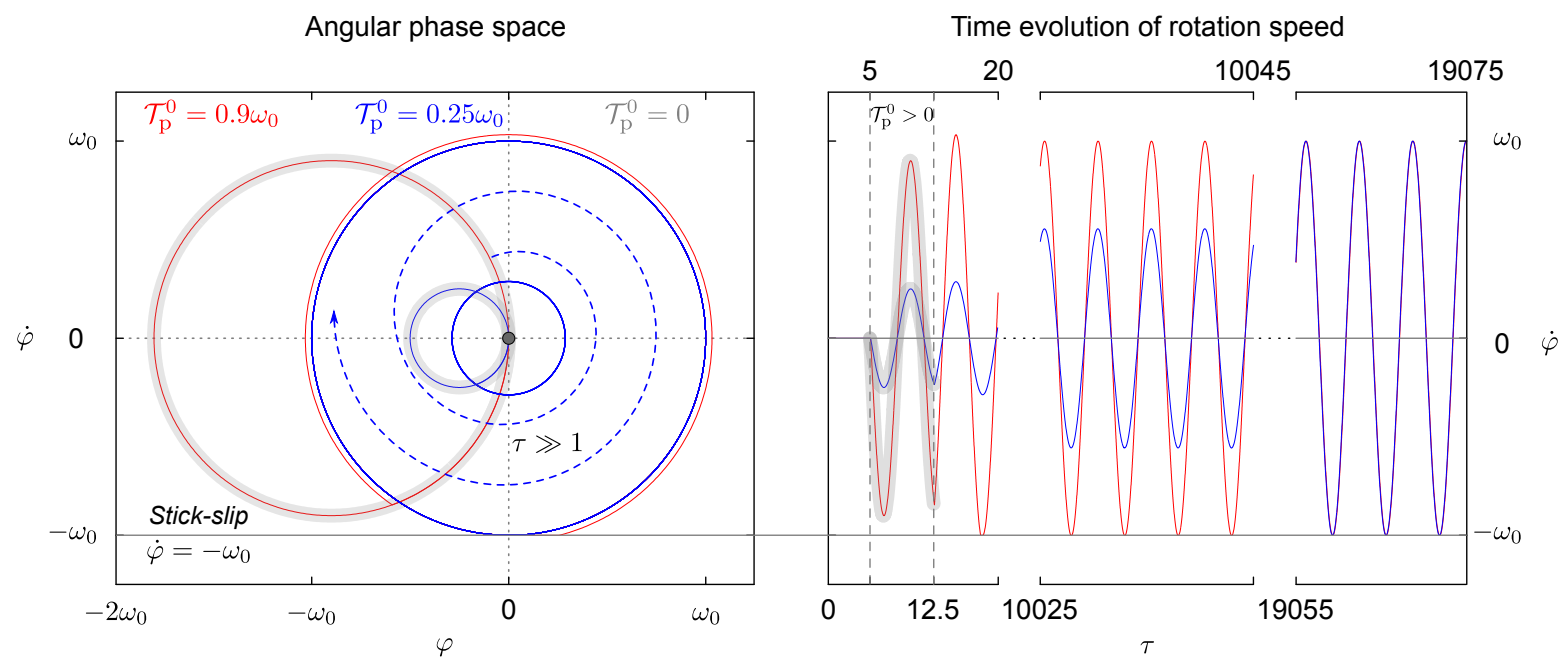

Figure 7: The occurrence of external perturbations while drilling may trigger unstable behaviors. However, for slow configurations, e.g., $\left(\omega_{0}, n, \psi, \alpha_{0}\right)=(20,6,100,0.1)$, the magnitude of the perturbations is critical to the observed regime of motion: moderate perturbations lead to a quasiperiodic oscillatory response around steady drilling (blue) that ultimately converges toward a stick-slip limit cycle, limit cycle to which the trajectory converges rapidly in case of large perturbations (red). The left plot depicts the projection of the trajectory in the angular space while the right one shows the evolution of the angular velocity with time. During the application of the external perturbation (whatever its magnitude), the angular dynamics experiences a projected circular trajectory in its phase space; see the shaded segments along the curves.

This transient analysis also confirms the necessity of taking the delay dynamics into account when assessing the stability of steady drilling. The steady-state response is, indeed, unstable for rotation speeds above the critical one, $\omega_{0}>\omega_{c}$, contrary to what is predicted by a stability analysis based on the linearized equations (24) or (25).

To make a parallel between the model and a realistic drilling situation, we consider the deep drilling system depicted in Figure 1, with the characteristics given in Table 1. Such a configuration is typical of the drilling conditions encountered by the oil and gas industry.

Building on these parameters, it is straightforward to compute the reference scales and dimensionless numbers required by the model definition. These are listed at the bottom of Table 1 . Noting the scale separation between $\alpha_{0}$ and $\psi$, the approximation $\omega_{c}^{0}$ to the critical rotation speed $\omega_{c}$ can be used. However, it is useful to convert equation (41) for $\omega_{c}^{0}$ into an explicit expression for the dimensional critical rotation speed $\Omega_{c}^{0}=\omega_{c}^{0} / t_{*}$ in terms of the physical parameters of the system, i.e.,

$$
\Omega_{c}^{0}=\sqrt{\frac{8 \zeta \varepsilon a}{n M}}=112 \mathrm{RPM}
$$

for the particular example under consideration.

Interestingly, the critical rotation speed at which the system switches from the fast instability regime to the slow one depends, within the framework of the RGD model, on a very limited set of parameters, namely: the interface parameter $\zeta$, the intrinsic specific energy $\varepsilon$, the bit radius $a$, the number of blades $n$ on the bit, and the mass $M$ of the BHA. Its apparent independence of the weight on bit and of the bit bluntness state results from (i) the scale difference between the system number $\psi$ and $\alpha_{0}\left(\psi \gg \alpha_{0}\right)$ that enables the simplification of the approximation of $\Omega_{c}$, and (ii) the linearization of the governing equations that removes any explicit dependency on parameter $\lambda$. The neighborhood of 

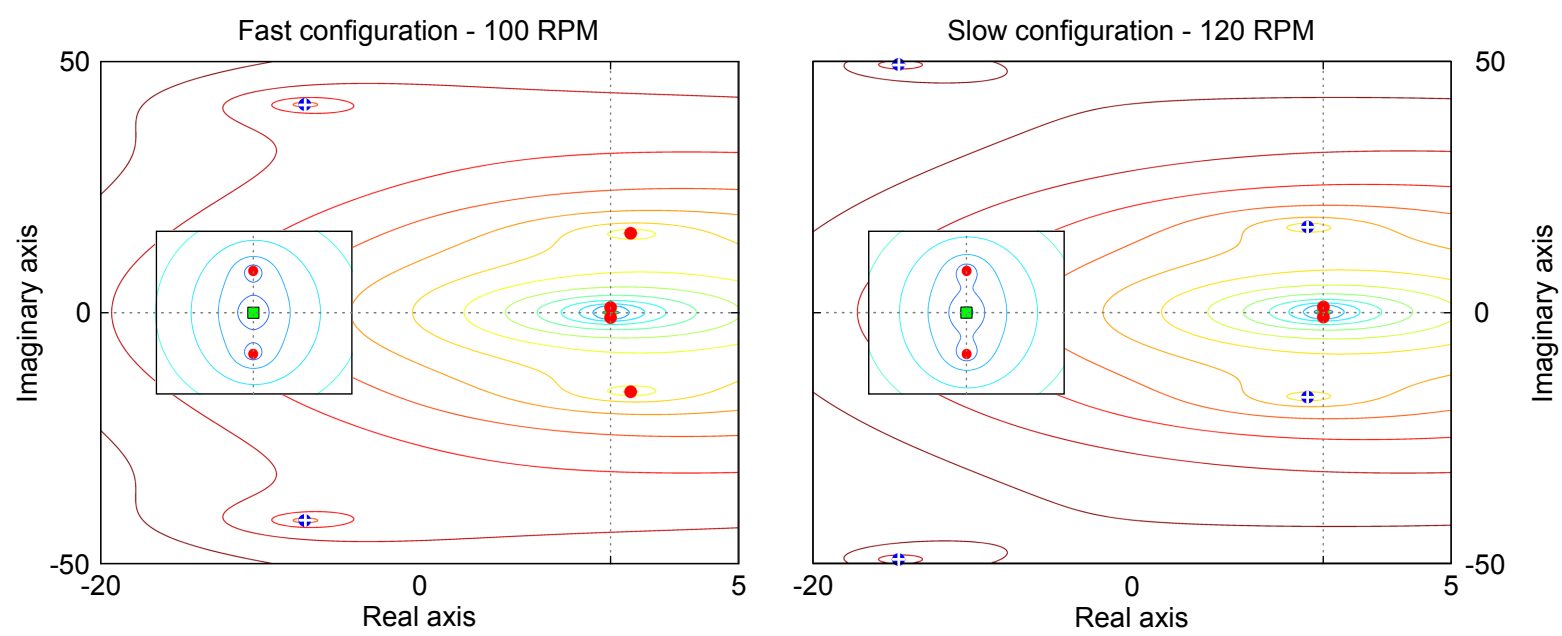

Figure 8: According to the model, in given drilling conditions, adjusting the rotation speed at the rig is likely to change the dynamics of the drilling process, as increasing it can bring the system from a fast to a slow instability regime. The representations above depict the locations in the complex plane of the system poles for two velocities $\Omega_{0}=100$ RPM (left) and $\Omega_{0}=120$ RPM (right) below and above the critical speed estimated at $\Omega_{c}^{0}=112$ RPM. Parameters of Table 1 have been used. White crossed markers denote poles with a negative real part, red disks the ones with a positive real part and the green squares those with zero real part, respectively. Level-set curves reflect the magnitude of the characteristic function $|P(s)|$. The roots of the characteristic equation have been computed using Matlab toolbox DDE-BIFTOOL [36]. The respective insets represent the neighborhood of the origin of the complex plane.

the steady-state solution in which the linearized equations and, a fortiori, the stability analysis hold does nonetheless depend on these parameters. The larger $\alpha_{0} \sim \mathcal{W}_{0}-\lambda$, the larger this neighborhood and the region of validity of the present analysis.

Following our developments, it is thus expected that operating the drilling rig at velocities below this threshold leads to an unstable process, prone to detrimental regimes such as bit-bouncing or stick-slip, whereas operating it above the critical velocity would mitigate the risk of the bit experiencing undesired vibration regimes. Figure 8 represents the poles associated with the example configuration for $\Omega_{0}=100 \mathrm{RPM}<\Omega_{c}^{0}$ (left) and $\Omega_{0}=120 \mathrm{RPM}>$ $\Omega_{c}^{0}$ (right). It confirms that the lower velocity configuration corresponds to a fast instability regime whereas the larger velocity one is to a slow instability regime.

Figure 9 further explores the stability properties of the system for the two rotation speeds. It represents a measure of the drilling process robustness to the perturbation of the sort defined by equation (43) by showing the type of motion the system converges to within $400 t_{*}=188$ seconds. Simulations are stopped once bit-boucing or stickslip is detected. The blue, light blue, yellow and red colors respectively denote a quasiperiodic oscillatory motion of the type illustrated in Figure 7, the convergence to a stick-slip limit cycle, the occurrence of backward rotation (due to a too long applied perturbation torque), and the onset of bit-bouncing. For the kind of perturbation that has been considered, the results confirm the prediction of the stability analysis. While any small perturbation triggers bit-bouncing instabilities for the fast configuration, larger and longer ones are required for an unstable drilling regime to be detected with the slow configuration.

Although the above results are specific to a particular family of exogenous perturbations, the stability analysis 
Table 1: List of the parameter values utilized for the example of Section 5.

\begin{tabular}{|c|c|c|c|}
\hline Parameter & Symbol & Value & Unit \\
\hline Weight on bit & $W_{0}$ & 15 & $\mathrm{kN}$ \\
\hline Rotation speed & $\Omega_{0}$ & $\{100,120\}$ & RPM \\
\hline Drill pipe outer radius & $r_{p, o}$ & $2^{1 / 2}$ & in \\
\hline Drill pipe inner radius & $r_{p, i}$ & $2^{1 / 8}$ & in \\
\hline Drill pipe length & $L_{p}$ & 2000 & $\mathrm{~m}$ \\
\hline BHA outer radius & $r_{b, o}$ & 3 & in \\
\hline BHA inner radius & $r_{b, i}$ & $1^{1 / 8}$ & in \\
\hline BHA length & $L_{b}$ & 250 & $\mathrm{~m}$ \\
\hline Steel shear modulus & $G$ & 77 & $\mathrm{GPa}$ \\
\hline Steel density & $\rho$ & 8000 & $\mathrm{~kg} / \mathrm{m}^{3}$ \\
\hline Drill pipe rotary inertia & $J_{p}=\frac{1}{2} \pi\left(r_{p, o}^{4}-r_{p, i}^{4}\right)$ & $1.22 \cdot 10^{-5}$ & $\mathrm{~m}^{4}$ \\
\hline Drill pipe torsional stiffness & $C=\frac{G J_{p}}{L_{p}}$ & 470 & $\mathrm{~N} \mathrm{~m}$ \\
\hline BHA mass & $M=\pi \rho\left(r_{b, o}^{2}-r_{b, i}^{2}\right) L_{b}$ & 31.35 & $\mathrm{~T}$ \\
\hline BHA inertia & $I=\frac{1}{2} M\left(r_{b, o}^{2}+r_{b, i}^{2}\right)$ & 103.8 & $\mathrm{~kg} \mathrm{~m}^{2}$ \\
\hline Bit radius & $a$ & 4.25 & in \\
\hline Bit geometry parameter & $\gamma$ & 1 & 1 \\
\hline Total wear flat length & $\ell$ & $1.8 \cdot 10^{-3}$ & $\mathrm{~m}$ \\
\hline Rock specific strength & $\varepsilon$ & 60 & $\mathrm{MPa}$ \\
\hline Bit/rock contact stress & $\sigma$ & 60 & $\mathrm{MPa}$ \\
\hline Cutting force inclination & $\zeta$ & 0.5 & 1 \\
\hline Bit/rock friction coefficient & $\mu$ & 0.6 & 1 \\
\hline Timescale & $t_{*}$ & 0.47 & s \\
\hline Length scale & $d_{*}$ & $1.34 \cdot 10^{-3}$ & $\mathrm{~m}$ \\
\hline Number of blades & $n$ & 6 & 1 \\
\hline Frictional torque parameter & $\beta$ & 0.3 & 1 \\
\hline Bluntness parameter & $\lambda$ & 2.68 & 1 \\
\hline System parameter & $\psi$ & 22.82 & 1 \\
\hline Rotation speed & $\omega_{0}$ & $\{4.92,5.91\}$ & 1 \\
\hline Steady depth of cut per radian & $\alpha_{0}$ & 0.12 & 1 \\
\hline
\end{tabular}



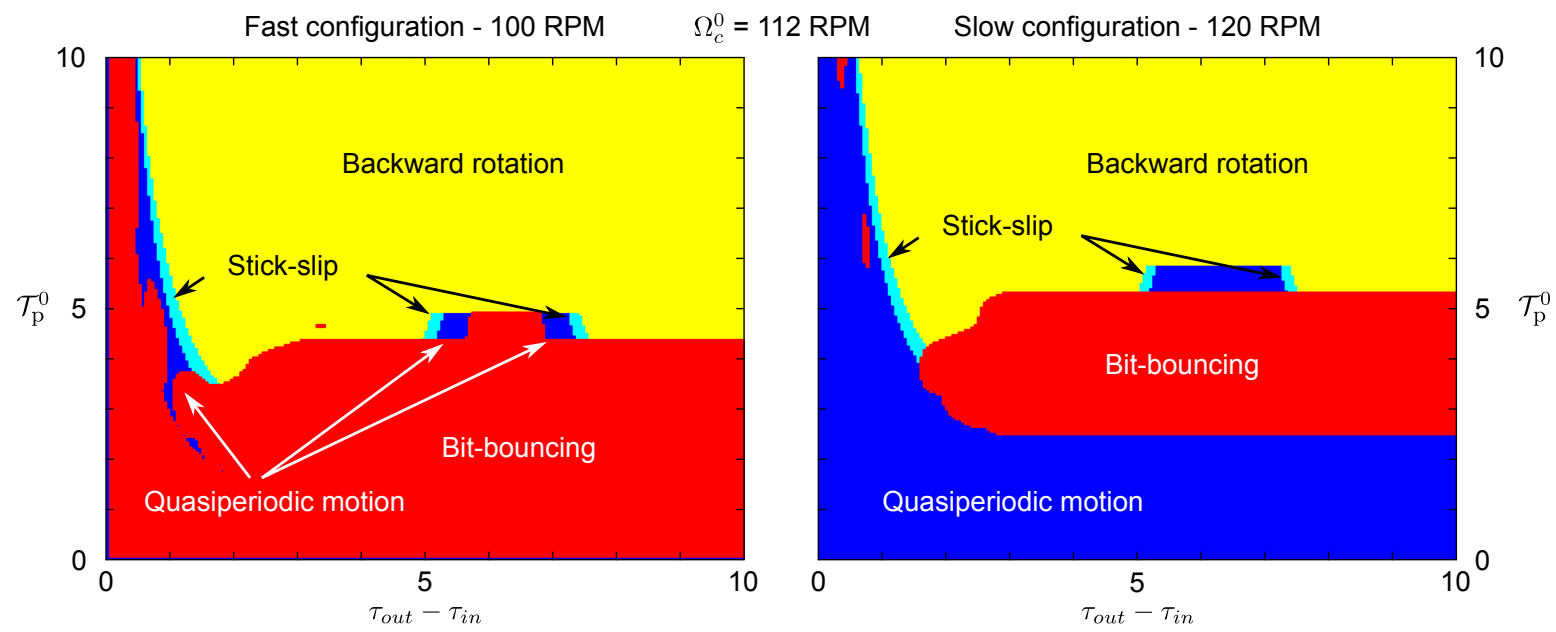

Figure 9: Reaction to a perturbation of type (43) in the case of a fast or slow configuration. Blue: the system experiences oscillatory response that has not converged to a dysfunction regime after $400 t_{*}=188$ seconds. Light blue: the system has converged to a stick-slip limit cycle within $400 t_{*}=188$ seconds. Red: bit-bouncing has been detected within $400 t_{*}=188$ seconds. Yellow: Backward rotation has been detected within $400 t_{*}=188$ seconds (consequence of a too long applied perturbation). Clearly, the slow configuration is less sensitive to perturbations (see perturbation magnitude required to set off bit-bouncing).

remains general. Its validity with respect to the actual drilling process is only contingent upon the adequacy of the RGD model and also the evaluation of its parameters.

The adequacy of the model has been discussed in the literature. Germay et al. [12] compared the RGD model with a finite element representation of the drillstring using rod elements. They concluded that the model is representative of the process, as long as the drillstring behaves like a torsional pendulum; that is, provided only the first torsional mode of the structure is excited during the vibrating process. This is dependent on the drillstring geometry and the operating conditions but nonetheless proves a good approximation in many circumstances. Variants of the RGD model have also been studied by Besselink et al. [13], and Nandakumar and Wiercigroch [14]. They considered a velocity rather than a force boundary condition on the axial degree of freedom at the rig. Accordingly, they introduced a linear spring in the axial direction, to account for the elasticity of the drillstring as well as structural damping. They also showed that stability of the steady-state solution is guaranteed if there is sufficient structural damping. We note, however, that the actual nature of the axial boundary condition at the rig is closer to a force control by design of the hoisting system and that the achieved penetration rate is a consequence of the applied force and not an input at the rig. Also, we understand that most dissipation takes place at the bit/rock interface, through the penetration of the bit in the rock. We therefore do not expect structural damping to fundamentally change the stability of steady drilling. At best, damping will contribute to alleviating bit-bouncing and stick-slip oscillations or delay their apparition.

Furthermore, the dimensional expression of the critical velocity (44) can be of inspiration in bit and BHA designs. As the critical velocity is inversely proportional to the square root of the BHA mass and the number of blades, we expect that, for a given rock formation and well diameter, a heavier BHA and a bit with numerous PDC cutters organized in blades will contribute toward the mitigation of stick-slip oscillations and other instabilities. 


\section{Conclusions}

This paper has focused on the linear stability analysis of a model of a deep drilling system originally introduced by Richard et al. [1,2] to study self-excited vibrations that could degenerate into stick-slip oscillations and bit-bouncing. The RGD model essentially reduces to two coupled state-dependent delay differential inclusions that govern the evolution of the axial and angular perturbations to the bit stationary motion.

This analysis has confirmed that the trivial solution characterized by a constant penetration rate and constant rotation speed of the bit is indeed unstable at any prescribed angular velocity $\Omega_{0}$, without consideration of any dissipation other than the rate-independent one that takes place at the bit/rock interface during drilling. However, we have shown that there exists in fact two regimes of instability, slow and fast, with a sharp transition taking place at a critical angular velocity $\Omega_{c}$. The slow regime $\left(\Omega_{0}>\Omega_{c}\right)$ is characterized by an extremely slow growth of the torsional oscillations, while the fast regime $\left(\Omega_{0}<\Omega_{c}\right)$ is associated with a rapid growth of the axial perturbations. For parametric configurations associated with the slow regime, the system behaves almost like a marginally stable system and its response is strongly influenced by external perturbations that could be encountered while drilling a rock formation, which are the cause of drilling regime transitions. For such drilling configurations, steady drilling coexists with almost periodic motion and potentially stick-slip oscillations, bit-bouncing or anti-resonance.

For typical field conditions, $\Omega_{c}$ can be approximated by the critical velocity computed by neglecting the delay dynamics, $\Omega_{c}^{0}$, which is a monomial function of a limited number of parameters; namely, the interface parameter $\zeta$, the intrinsic specific energy $\varepsilon$, the bit radius $a$, the number of blades $n$ on the bit, and the mass $M$ of the BHA. Though the weight on bit and the bit bluntness state do not explicitly enter the expression of the critical velocity approximation, they play a role in the definition of the domain of validity of the presented stability analysis; the larger the penetration rate at steady state, the larger this domain.

These findings have been illustrated by an application example. It shows that knowing the current configuration of the drilling system, i.e., the model parameters, proper adjustment of the rotational speed imposed at the rig rotary table can help mitigate the drilling instabilities encountered in practice, e.g., stick-slip vibrations.

\section{References}

[1] T. Richard, C. Germay, E. Detournay, Self-Excited Stick-Slip Oscillations of Drill Bits, Comptes Rendus Mécanique, French Academy of Sciences 332 (2004) 619-626. doi:10.1016/j.crme.2004.01.016.

[2] T. Richard, C. Germay, E. Detournay, A simplified model to explore the root cause of stick-slip vibrations in drilling systems with drag bits, Journal of Sound and Vibration 305 (3) (2007) 432-456. doi:10.1016/j.jsv.2007.04.015.

[3] D. R. Pavone, J. P. Desplans, Application of high sampling rate downhole measurements for analysis and cure of stick-slip in drilling, SPE 28324 (1994).

[4] J. F. Brett, The genesis of torsional drillstring vibrations, SPE Drilling Engineering (1992) 168-174.

[5] J. Jansen, L. van den Steen, E. Zachariasen, Active damping of torsional drillstring vibrations with a hydraulic top drive, SPE Drilling \& Completion (1995) 250-254.

[6] V. A. Palmov, E. Brommundt, A. K. Belyaev, Stability analysis of drillstring rotation, Dynamics and Stability of Systems 10 (2) (1995) 99-110. doi:10.1080/02681119508806197.

[7] R. W. Tucker, C. Wang, An integrated model for drill-string dynamics, Journal of Sound and Vibration 224 (1) (1999) $123-165$. doi:10.1006/jsvi.1999.2169.

[8] N. Challamel, Rock destruction effect on the stability of a drilling structure, Journal of Sound and Vibration 233 (2) (2000) $235-254$. doi:10.1006/jsvi.1999.2811. 
[9] A. G. Balanov, N. B. Janson, P. V. E. McClintock, R. W. Tucker, C. H. T. Wang, Bifurcation analysis of a neutral delay differential equation modelling the torsional motion of a driven drill-string, Chaos, Solitons \& Fractals 15 (2) (2003) 381-394. doi:10.1016/S0960-0779(02)001054.

[10] A. S. Yigit, A. P. Christoforou, Stick-slip and bit-bounce interaction in oil-well drillstrings, ASME Journal of Energy Resources Technology 128 (4) (2006) 268-274. doi:10.1115/1.2358141.

[11] E. Detournay, P. Defourny, A phenomenological model of the drilling action of drag bits, International Journal of Rock Mechanics and Mining Sciences 29 (1) (1992) 13-23.

[12] C. Germay, V. Denoel, E. Detournay, Multiple mode analysis of the self-excited vibrations of rotary drilling systems, Journal of Sound and Vibration 325 (1-2) (2009) 362-381. doi:10.1016/j.jsv.2009.03.017.

[13] B. Besselink, N. van de Wouw, H. Nijmeijer, A semi-analytical study of stick-slip oscillations in drilling systems, Journal of Computational and Nonlinear Dynamics 6 (2) (2011) 021006-1-9. doi:10.1115/1.4002386.

[14] K. Nandakumar, M. Wiercigroch, Stability analysis of a state dependent delayed, coupled two DOF model of drill-string vibration, Journal of Sound and Vibration 332 (10) (2013) 2575-2592. doi:10.1016/j.jsv.2012.12.020.

[15] K. Nandakumar, M. Wiercigroch, Galerkin projections for state-dependent delay differential equations with applications to drilling, Applied Mathematical Modelling 37 (4) (2013) 1705-1722. doi:10.1016/j.apm.2012.04.038.

[16] T. Insperger, G. Stépán, J. Turi, State-dependent delay model for regenerative cutting processes, in: Proceedings of Fifth EUROMECH Nonlinear Dynamics Conference, ENOC 2005, Eindhoven, Netherlands, 2005, pp. 1124-1129.

[17] T. Insperger, G. Stépán, J. Turi, State-dependent delay in regenerative turning processes, Nonlinear Dynamics 47 (2007) $275-283$. doi:10.1007/s11071-006-9068-2.

[18] T. Insperger, D. Barton, G. Stépán, Criticality of Hopf bifurcation in state-dependent delay model of turning processes, International Journal of Non-Linear Mechanics 43 (2008) 140-149. doi:10.1016/j.ijnonlinmec.2007.11.002.

[19] C. Germay, N. van de Wouw, H. Nijmeijer, R. Sepulchre, Nonlinear drillstring dynamics analysis, SIAM Journal Applied Dynamical Sytems 8 (2) (2009) 527-553. doi:10.1137/060675848.

[20] R. I. Leine, D. H. van Campen, W. J. G. Keultjes, Stick-slip whirl interaction in drillstring dynamics, Journal of Vibration and Acoustics 124 (2002) 209-220. doi:10.1115/1.1452745.

[21] E. Detournay, T. Richard, M. Shepherd, Drilling response of drag bits: Theory and experiment, International Journal of Rock Mechanics and Mining Sciences 45 (8) (2008) 1347-1360. doi:10.1016/j.ijrmms.2008.01.010.

[22] T. Richard, F. Dagrain, E. Poyol, E. Detournay, Rock strength determination from scratch tests, Engineering Geology 147-148 (2012) 91-100. doi:10.1016/j.enggeo.2012.07.011.

[23] R. I. Leine, N. van de Wouw, Stability and Convergence of Mechanical Systems with Unilateral Constraints, 1st Edition, Springer Publishing Company, Incorporated, 2008.

[24] F. Hartung, J. Turi, Linearized stability in functional differential equations with state-dependent delays, International Conference on Dynamical Systems and Differential Equations, 2000.

[25] F. Hartung, J. Turi, Stability in a class of functional differential equations with state-dependent delays, Qualitative Problems for Differential Equations and Control Theory, Word Scientific, 1995, pp. 15-31.

[26] T. Luzyanina, K. Engelborghs, D. Roose, Numerical bifurcation analysis of differential equations with state-dependent delay, International Journal of Bifurcation and Chaos 11 (3) (2001) 737-753. doi:10.1142/S0218127401002407.

[27] F. Hartung, Linearized stability in periodic functional differential equations with state-dependent delays, Journal of Computational and Applied Mathematics 174 (2) (2005) 201-211. doi:10.1016/j.cam.2004.04.006.

[28] T. Erneux, Applied Delay Differential Equations, 1st Edition, Springer Science+Business Media, LLC, 2009.

[29] T. Carr, R. Haberman, T. Erneux, Delay-periodic solutions and their stability using averaging in delay-differential equations, with applications, Physica D: Nonlinear Phenomena 241 (18) (2012) 1527-1531. doi:10.1016/j.physd.2012.06.001.

[30] A. M. Krall, On the real parts of zeros of exponential polynomials, Bulletin of the American Mathematical Society 70 (2) (1964) 291-292.

[31] G. Stépàn, Retarded Dynamical System, Longman, London, 1989.

[32] K. Nandakumar, M. Wiercigroch, C. Pearson, Bit-bounce and stick-slip in drill-string dynamics, in: M. Wiercigroch, G. Rega (Eds.), Proceedings of the IUTAM symposium on Nonlinear dynamics for advanced technologies in engineering design, 2010.

[33] D. Bernstein, G. Coon, On the zeros of a class of exponential polynomials, Journal of Mathematical Analysis and Applications 11 (1965) 205-212.

[34] D. E. Gilsinn, Estimating critical hopf bifurcation parameters for a second-order delay differential equation with application to machine tool chatter, Nonlinear Dynamics 30 (2002) 103-154. doi:10.1023/A:1020455821894

[35] R. P. H. Faassen, N. van de Wouw, J. A. J. Oosterling, H. Nijmeijer, Prediction of regenerative chatter by modeling and analysis of high-speed milling, International Journal of Machine Tool and Manufacture 43 (2003) 1437-1445. doi:10.1016/S0890-6955(03)00171-8.

[36] K. Engelborghs, T. Luzyanina, G. Samaey, DDE-BIFTOOL v. 2.00: a Matlab package for bifurcation analysis of delay differential equations, Tech. rep., K.U.Leuven (2001).

[37] A. Depouhon, Stability of TAZ apparatus: A drilling equivalent, Master's thesis, Univ. Minnesota (2007).

[38] L. Ledgerwood, O. Hoffmann, J. Jain, C. El Hakam, C. Herbig, R. Spencer, Downhole vibration measurement, monitoring and modeling reveal stick-slip as a primary cause of pdc bit damage in today's applications, SPE 134488, 2010.

\section{Appendix A. Derivation of the Characteristic Equation}

Let the state vector $\mathbf{x}$ be defined by

$$
\mathbf{x}(\tau)=[u(\tau), \dot{u}(\tau), \varphi(\tau), \dot{\varphi}(\tau)]^{T},
$$


To derive the characteristic equation associated with the drilling model and assess the stability of its steady state, we rewrite the linearized delay differential equations (28) as a system of first order differential equations. In matrix form, this system reads

$$
\dot{\mathbf{x}}(\tau)=\mathbf{A} \mathbf{x}(\tau)+\breve{\mathbf{A}} \mathbf{x}\left(\tau-\tau_{n 0}\right)
$$

where $\mathbf{A}$ and $\breve{\mathbf{A}}$ are the matrices containing the coefficients of the current and delayed state variables, respectively

$$
\mathbf{A}=\left[\begin{array}{cccc}
0 & 1 & 0 & 0 \\
-n \psi & 0 & n \psi \alpha_{0} & 0 \\
0 & 0 & 0 & 1 \\
-n & 0 & n \alpha_{0}-1 & 0
\end{array}\right], \quad \breve{\mathbf{A}}=\left[\begin{array}{cccc}
0 & 0 & 0 & 0 \\
n \psi & 0 & -n \psi \alpha_{0} & 0 \\
0 & 0 & 0 & 0 \\
n & 0 & -n \alpha_{0} & 0
\end{array}\right] .
$$

The characteristic equation (29), $P(s)=0$, is then obtained by setting to zero the determinant of the characteristic function

$$
\Delta(s)=s \mathbf{I}-\mathbf{A}-\breve{\mathbf{A}} \mathrm{e}^{-s \tau_{n 0}},
$$

obtained by either postulating an exponential Ansatz in (A.2) or Laplace transforming it.

The same procedure applies to calculate $P^{0}(s)$ given in equation (31). The corresponding matrices $\mathbf{A}$ and $\breve{\mathbf{A}}$ are given by (A.3) with $\alpha_{0}=0$.

\section{Appendix B. Crossing Direction of the Imaginary Axis by the System Poles}

To further understand the root locus of the system poles as the steady-state delay is varied, we compute the crossing directions at its intersections with the imaginary axis. We do so by computing the total derivative of the characteristic function with respect to the delay at a crossing point $s_{c}= \pm \mathrm{j} \varpi_{*}$, namely

$$
\frac{\partial s_{c}}{\partial \tau_{n 0}}=-\frac{\partial P / \partial \tau_{n 0}}{\partial P / \partial s}
$$

hence,

$$
\frac{\partial s_{c}}{\partial \tau_{n 0}}=-\frac{n s\left(\psi+s_{c}^{2}\left(\psi-\alpha_{0}\right)\right)}{n s_{c}\left(\psi-\alpha_{0}\right)\left(s_{c} \tau_{n 0}-2+2 \mathrm{e}^{\tau_{n 0} s_{c}}\right)-n \psi \tau_{n 0}-2 s_{c} \mathrm{e}^{\tau_{n 0} s_{c}}\left(1+2 s_{c}^{2}\right)} .
$$

First, we consider the crossings taking place at $\left(s_{c}, \tau_{n 0}\right)=( \pm \mathrm{j}, 2 m \pi)$, with $m \in \mathbb{Z}^{+}$. Evaluating (B.1) at this critical point, we find

$$
\frac{\partial s_{c}}{\partial \tau_{n 0}}=\frac{n \alpha_{0}}{2} \frac{1 \mp \mathrm{j} m \pi n \alpha_{0}}{1+\left(m \pi n \alpha_{0}\right)^{2}}
$$

from which it readily appears that

$$
\operatorname{sign}\left(\operatorname{Re}\left(\frac{\partial s_{c}}{\partial \tau_{n 0}}\right)\right)=1 \quad \text { and } \quad \operatorname{sign}\left(\operatorname{Im}\left(\frac{\partial s_{c}}{\partial \tau_{n 0}}\right)\right)=\mp 1
$$


as long as $\alpha_{0}>0$. Thus, roots crossing at $s_{c}= \pm \mathrm{j}$ do so from the LHS to the RHS and from the poles $( \pm \mathrm{j} \infty)$ to the equator $(0 \mathrm{j})$ by symmetry of the system. The case $\alpha_{0}=0$ is equivalent to the uncoupling of the axial and torsional dynamics, in which case the torsional poles are insensitive to variations of the delay and remain at $s_{t}= \pm \mathrm{j}$.

We then apply the same reasoning at $\left(s_{c}, \tau_{n 0}\right)=\left( \pm \mathrm{j} \varpi_{*}^{ \pm},(2 m-1) \pi / \varpi_{*}^{ \pm}\right)$, with $m \in \mathbb{Z}^{+}$. We get

$$
\frac{\partial s_{c}}{\partial \tau_{n 0}}=\frac{n \varpi_{*}^{ \pm, 2}\left(\psi-\left(\psi-\alpha_{0}\right)\left(\varpi_{*}^{ \pm}\right)^{2}\right)}{2\left(\varpi_{*}^{ \pm}\right)^{2}\left(1+2 n\left(\psi-\alpha_{0}\right)-2\left(\varpi_{*}^{ \pm}\right)^{2}\right)+\mathrm{j}(2 m+1) \pi n\left(\psi-\left(\psi-\alpha_{0}\right)\left(\varpi_{*}^{ \pm}\right)^{2}\right)},
$$

from which we have

$$
\begin{aligned}
& \operatorname{sign}\left(\operatorname{Re}\left(\frac{\partial s_{c}}{\partial \tau_{n 0}}\right)\right)=\operatorname{sign}\left(\psi-\left(\psi-\alpha_{0}\right)\left(\varpi_{*}^{ \pm}\right)^{2}\right) \cdot \operatorname{sign}\left(1+2 n\left(\psi-\alpha_{0}\right)-2\left(\varpi_{*}^{ \pm}\right)^{2}\right), \\
& \operatorname{sign}\left(\operatorname{Im}\left(\frac{\partial s_{c}}{\partial \tau_{n 0}}\right)\right)=\mp\left(\operatorname{sign}\left(\psi+\left(\alpha_{0}-\psi\right)\left(\varpi_{*}^{ \pm}\right)^{2}\right)\right)^{2} .
\end{aligned}
$$

It can be shown that, under the constraint $0<\alpha_{0}<\alpha_{0}^{-}$, the sign of term $\psi+\left(\alpha_{0}-\psi\right)\left(\varpi_{*}^{ \pm}\right)^{2} \lessgtr 0$ follows the magnitude of $n \psi \lessgtr 1 / 2$ and that the sign of $-\left(1-2\left(\varpi_{*}^{ \pm}\right)^{2}-2 n\left(\alpha_{0}-\psi\right)\right)$ corresponds to that of the $\varpi_{*}^{ \pm}$root superscript. We therefore have that crossings at $s_{c}= \pm \mathrm{j} \varpi_{*}^{+}$are from LHS to RHS and those at $s_{c}= \pm \mathrm{j} \varpi_{*}^{-}$are from RHS to LHS when $n \psi>1 / 2$ and in the opposite direction when $n \psi<1 / 2$; plus, the poles are moving towards the equator during the crossings.

Furthermore, the order of the crossing points can be shown to depend on the magnitude of $n \psi$. If the configuration corresponds to region $\mathrm{I}_{a}(n \psi>1 / 2)$, we have $\varpi_{*}^{+}>\varpi_{*}^{-}>1$. However, the reverse identity holds if the system configuration is in region $\mathrm{I}_{b}(n \psi<1 / 2)$, namely $\varpi_{*}^{-}<\varpi_{*}^{+}<1$. The root loci associated with regions $\mathrm{I}_{a}$ and $\mathrm{I}_{b}$ are thus topologically non-equivalent.

Finally, we mention that the torsional poles leave $s_{c}= \pm \mathrm{j}$ orthogonally to the imaginary axis as the delay is increased from 0

$$
\frac{\partial s_{c}}{\partial \tau_{n 0}}=\left.\frac{n \alpha_{0}}{2}\right|_{\left(s_{c}, \tau_{n 0}\right)=( \pm \mathrm{j}, 0)}
$$

\section{Appendix C. Approximated Expression for the Real Part of the Unstable Slow Poles}

In Section 4.1, we have derived two approximations to the critical rotation speed $\omega_{c}$. These are given by equations (40) and (41) and approximate the transition speed by the one corresponding to the first crossing of the imaginary axis by axial poles, accounting or not for the dynamics of the delay. They are valid for parameters that reflect conditions for deep drilling systems. We refer to them as approximations 1 and 2, respectively.

Further exploiting the proximity of the angular poles to the imaginary axis, we estimate the location of the torsional poles, $s_{t}^{ \pm}$, by expanding to first-order the characteristic equation around $s= \pm \mathrm{j}$, location of the angular poles of the uncoupled system. This yields

$$
s_{t}^{ \pm} \simeq \pm \mathrm{j}+\left.n \alpha_{0} \frac{\Delta P(s)}{\left(P^{0}(s)-n \alpha_{0} \Delta P(s)\right)^{\prime}}\right|_{s= \pm \mathrm{j}},
$$



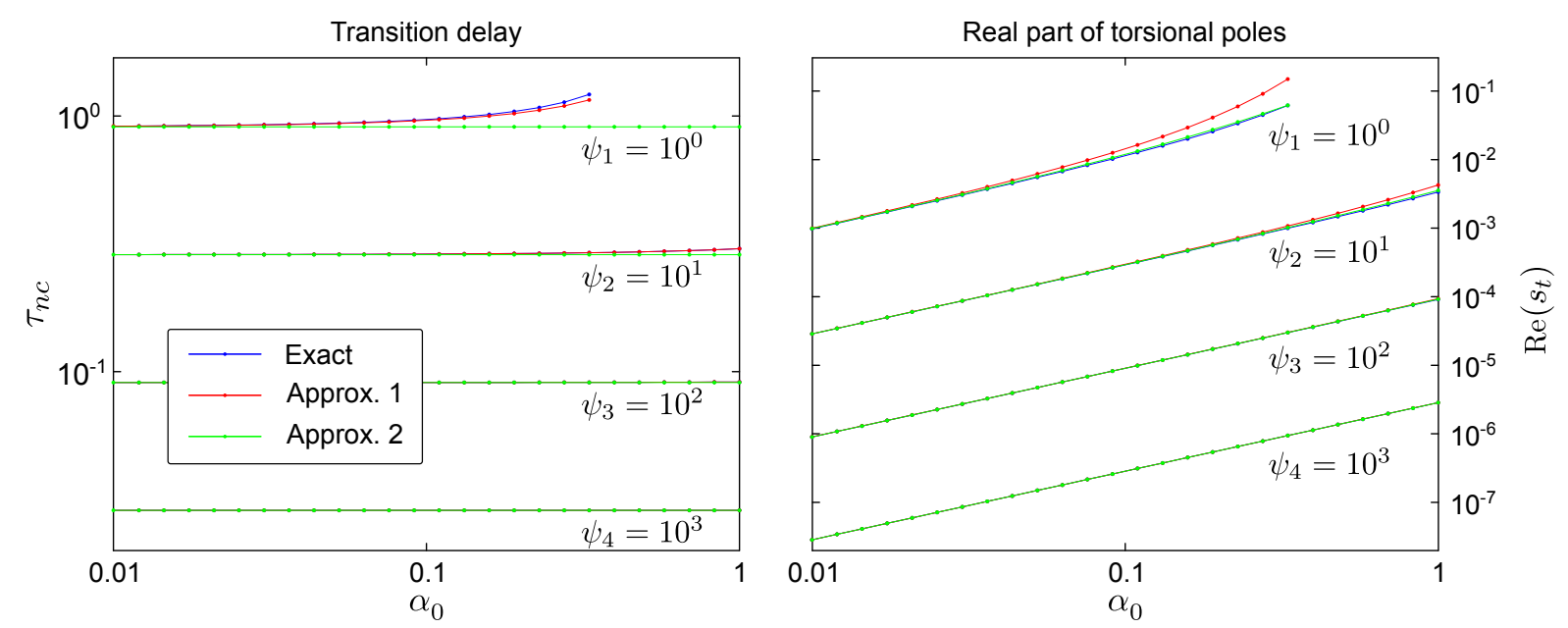

Figure C.1: Under the condition $\psi \gg \alpha_{0}$, the approximations of the transition delay (left) and of the real part of the torsional poles (right) show a good match with the exact values. The graphs are in log-log scale.

with

$$
\begin{aligned}
\Delta P( \pm \mathrm{j}) & =\mathrm{e}^{\mp \mathrm{j} \tau_{n c}}-1, \\
P^{0^{\prime}}( \pm \mathrm{j}) & = \pm 2 \mathrm{j}\left(n \psi\left(1-\mathrm{e}^{\mp \mathrm{j} \tau}\right)-1\right), \\
\Delta P^{\prime}( \pm \mathrm{j}) & = \pm 2 \mathrm{j}\left(1-\mathrm{e}^{\mp \mathrm{j} \tau}\right)-\tau_{n c} \mathrm{e}^{\mp \mathrm{j} \tau_{n c}},
\end{aligned}
$$

and $\tau_{n c}$ given by approximation 1, i.e., equation (39). Following the assumption of approximation 2 , i.e., $\psi \gg \alpha_{0}$, the approximation of the torsional poles further simplifies and their real part can be approximated by

$$
\operatorname{Re}\left(s_{t}^{ \pm}\right)=\frac{\tau_{n c}\left(n \alpha_{0}\right)^{2}\left(1-\cos \tau_{n c}\right)+2 n \alpha_{0} \sin \tau_{n c}}{4-8 n \psi+n^{2}\left(\left(\alpha_{0} \tau_{n c}\right)^{2}+8 \psi^{2}\right)+8 n \psi \cos \tau_{n c}(1-n \psi)+4 n \alpha_{0} \tau_{n c} \sin \tau_{n c}(1-n \psi)}
$$

with $\tau_{n c}$ defined in equation (34).

Comparison of the set of approximations 1 and 2 with the exact transition delay and the real part of the torsional poles are shown in Figure C. 1 for $\alpha_{0} \in[0.01,1]$ and four values of the parameter $\psi$. It is seen that the approximations provide a good estimation of the exact solution, in particular when $\psi \gg \alpha_{0}$. In that case, the transition delay is almost insensitive to $\alpha_{0}$ and the real part of the torsional pole increases linearly with $\alpha_{0}$. The approximation ceases to be valid when $\alpha_{0}$ becomes of the order of magnitude of $\psi$, precisely when $\alpha_{0}$ becomes larger than $\alpha_{0}^{-}(n, \psi)$, a parametric configuration that leads to a change of the root locus topology and an alteration of the number of crossing points on the imaginary axis (see Section 3.4 and Appendix D).

\section{Appendix D. Root locus of the System Poles in Complex Plane for Regions $\mathbf{I}_{b}$, II and III}

In Section 4.1, we have described the trajectory in the complex plane of the system poles when the steady-state delay is varied from 0 to positive values, for a configuration corresponding to deep drilling applications. This qualitative description does not hold, however, when considering parametric configurations outside the region $\mathrm{I}_{a}$ defined in 
Figure 3. It is the purpose of this section to qualitatively describe the root locus of the system poles upon variation of the delay, for regions $\mathrm{I}_{b}$, II, and III, as well as the limiting cases (except $\alpha_{0}=0$ that is equivalent to 0 penetration).

The graphs presented in the sequel are to be understood as follows:

- The initial configuration corresponds to the undelayed situation, i.e., $\tau_{n 0}=0$. Though this case is not physically relevant (it corresponds to an infinite rotation speed of the bit), it is mathematically speaking, and we use it as the starting point of our description.

- Upon monotonous increase of the steady-state delay from $\tau_{n 0}=0$, the system poles move in the complex plane. Their trajectory might intersect or become tangent to the imaginary axis at a certain number of locations. This number depends on the parametric configuration under scrutiny. All configurations present at least one pair of crossing points at $s_{c}= \pm \mathrm{j}$.

- Increasing the delay from 0 drives a pole from the origin to the LHS of the complex plane, whatever the parametric configuration. The pole that remains at the origin can be discarded from the stability analysis, for the steady-state response is defined up to a displacement offset.

- In the following figures, the origin of the trajectory is indicated by the ellipsis and the direction of motion is provided by an arrow at the end of the curve representing a pole trajectory. Torsional poles always originate at $s_{c}= \pm \mathrm{j}$ while axial ones originate in the LHS of the complex plane. We do not represent all pole trajectories, as there is an infinite number of them.

- Trajectories of the torsional poles are indicated using dashed lines while the ones of axial poles are represented using solid lines. In case the torsional poles may exhibit topologically different trajectories, they are superimposed on the same graph. They do however correspond to different parameter sets.

These qualitative descriptions are based on numerical investigations combined with algebraic developments. They are proposed as indicators of the different behaviors to be expected from the RGD model, should it be applied to parametric configurations other than related to deep drilling. As these configurations are not the main focus of this research, our investigations are not as complete as for the region $\mathrm{I}_{a}$, relevant to deep drilling configurations.

Appendix D.1. Region $\mathrm{I}_{b}:\left(n \psi, n \alpha_{0}\right) \in(0,1 / 2) \times\left(0, \alpha_{0}^{-}\right)$

Similarly to region $\mathrm{I}_{a}$, pole trajectories cross the imaginary axis at three pairs of conjugated locations, $s_{c}= \pm \mathrm{j}$ and $s_{c}= \pm \mathrm{j} \varpi_{*}^{ \pm}$, with $\varpi_{*}^{ \pm}$given by equation (38). However, we now have $\varpi_{*}^{-}<\varpi_{*}^{+}<1$, so that axial poles first cross the imaginary axis at $s_{c}= \pm \mathrm{j}$, then at $s_{c}= \pm \mathrm{j} \varpi_{*}^{+}$, and finally at $s_{c}= \pm \mathrm{j} \varpi_{*}^{-}$when the delay is increased from 0 . The crossing directions at $s_{c}= \pm \mathrm{j} \varpi_{*}^{ \pm}$are opposite to those of region $\mathrm{I}_{a}$, with a crossing from RHS to LHS at $s_{c}= \pm \mathrm{j} \varpi_{*}^{+}$, and from LHS to RHS at $s_{c}= \pm \mathrm{j} \varpi_{*}^{-}$. The critical delays at which crossings occur are given by equation (38) and $\tau_{m}=2 m \pi$, with $m \in \mathbb{Z}^{+}$.

Figure D.1 illustrates the root locus of the system poles. 


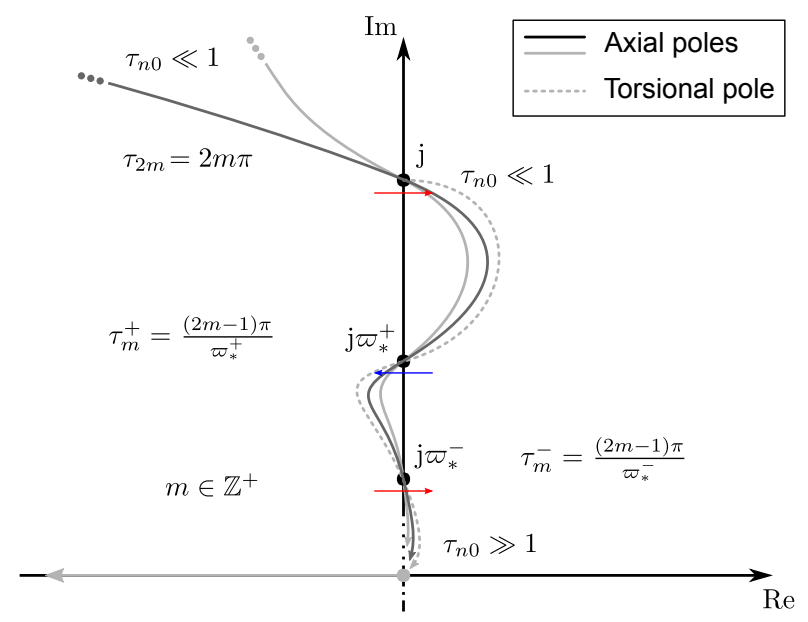

Figure D.1: Root locus of the system poles for parametric configurations in region $\mathrm{I}_{b}$. Pole trajectories intersect the imaginary axis at three locations. Both the axial and torsional poles converge to the origin of the complex plane.

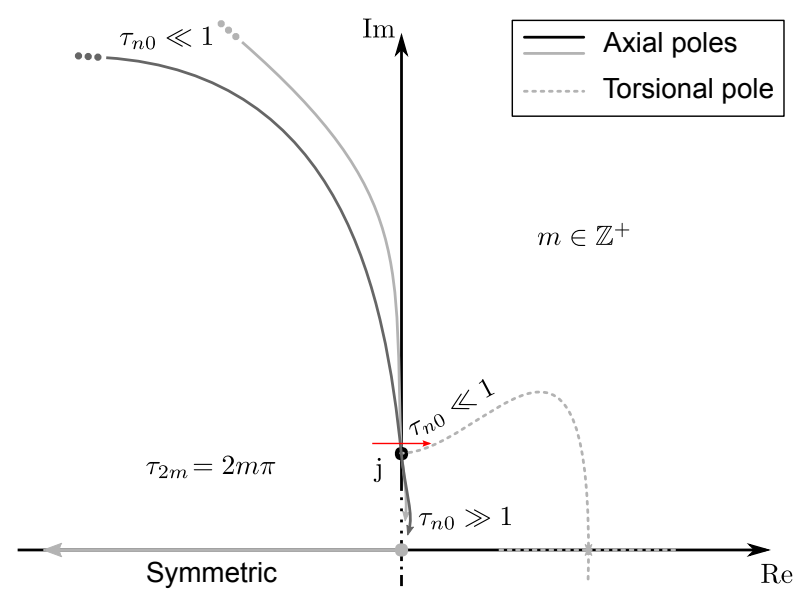

Figure D.2: Root locus of the system poles for parametric configurations in region II. Only one conjugated crossing point exists, at $s_{c}= \pm \mathrm{j}$.

Appendix D.2. Region II: $\left(n \psi, n \alpha_{0}\right) \in(0,+\infty) \times\left(n \alpha_{0}^{+},+\infty\right)$

For parametric configurations in region II, arguments $C_{1}$ and $C_{2}$ are respectively positive and negative, $C_{1}>0$ and $C_{2}<0$. Accordingly, the pulsations $\varpi_{*}^{ \pm}$defined in equations (38) are complex numbers and are not solutions of equations (35). The only crossing points are thus $s_{c}= \pm \mathrm{j}$ and the crossings take place at $\tau_{m}=2 m \pi$, with $m \in \mathbb{Z}^{+}$. Again, the torsional poles leave $s_{c}= \pm \mathrm{j}$ orthogonally to the imaginary axis when the delay is increased from zero. They govern the instability of the system that is unstable for any $\tau_{n 0}>0$.

Figure D.2 illustrates the root locus of the system poles.

Appendix D.3. Region III: $\left(n \psi, n \alpha_{0}\right) \in(0,+\infty) \times\left(n \alpha_{0}^{-}, n \alpha_{0}^{+}\right)$

In region III, arguments $C_{1}$ and $C_{2}$ are both negative, $C_{1}, C_{2}<0$, leading to complex pulsations $\varpi_{*}^{ \pm}$that are not solutions of equations (35). Similarly to region II, there is only one pair of conjugated crossing points at $s_{c}= \pm \mathrm{j}$, for 


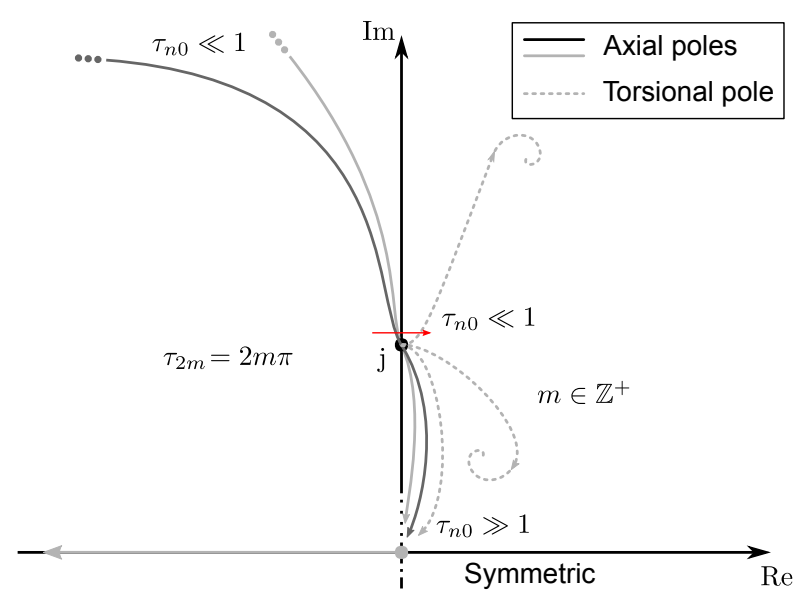

Figure D.3: Root locus of the system poles for parametric configurations in region III. Only one conjugated crossing point exists, at $s_{c}= \pm \mathrm{j}$. Depending on the magnitude of $n \psi$, the torsional poles either converge to the origin or to a focus point in the RHS of the complex plane. The graph displays three possible trajectories for the torsional poles (dashed lines). They are each specific to one parametric configuration.

critical delays $\tau_{m}=2 m \pi$, with $m \in \mathbb{Z}^{+}$. Depending on the magnitude of $n \psi$, our investigations have revealed that the torsional poles can follow trajectories of different natures. Either they converge to the origin of the complex plane, following a trajectory that remains close to the imaginary axis, or they converge to some focus point in the RHS of the complex plane, in the vicinity of which they remain once the delay is large enough. Instabilities are governed by the torsional poles. The system is unstable for any positive delay.

This is illustrated in Figure D.3 where three possible trajectories for the torsional poles are shown. They each occur for a different value of parameter $n \psi$.

Appendix D.4. Limit cases: $n \alpha_{0}=n \alpha_{0}^{ \pm}$

When $n \alpha_{0}=n \alpha_{0}^{-}$, pulsations $\varpi_{*}^{ \pm}$become equal, $\varpi_{*}^{+}=\varpi_{*}^{-}=\varpi_{*}$. As a consequence, the root locus becomes tangent to the imaginary axis at $s_{c}= \pm \mathrm{j} \varpi_{*}$, with a conjugated crossing point still located at $s_{c}= \pm \mathrm{j}$ where poles go from the LHS to the RHS of the complex plane. Depending on the magnitude of $n \psi \lessgtr 1 / 2$, we have $\varpi_{*} \lessgtr 1$, which affects the topology of the root locus, as depicted in Figure D.4. Again, the torsional poles either converge to the origin of the complex plane or to some focus point, depending on the magnitude of parameter $n \psi$. The steady-state response is unstable for any positive delay and is ruled by the torsional poles.

The limit case $n \alpha_{0}=n \alpha_{0}^{+}$yields a root locus similar to that of region III and is not repeated here. 

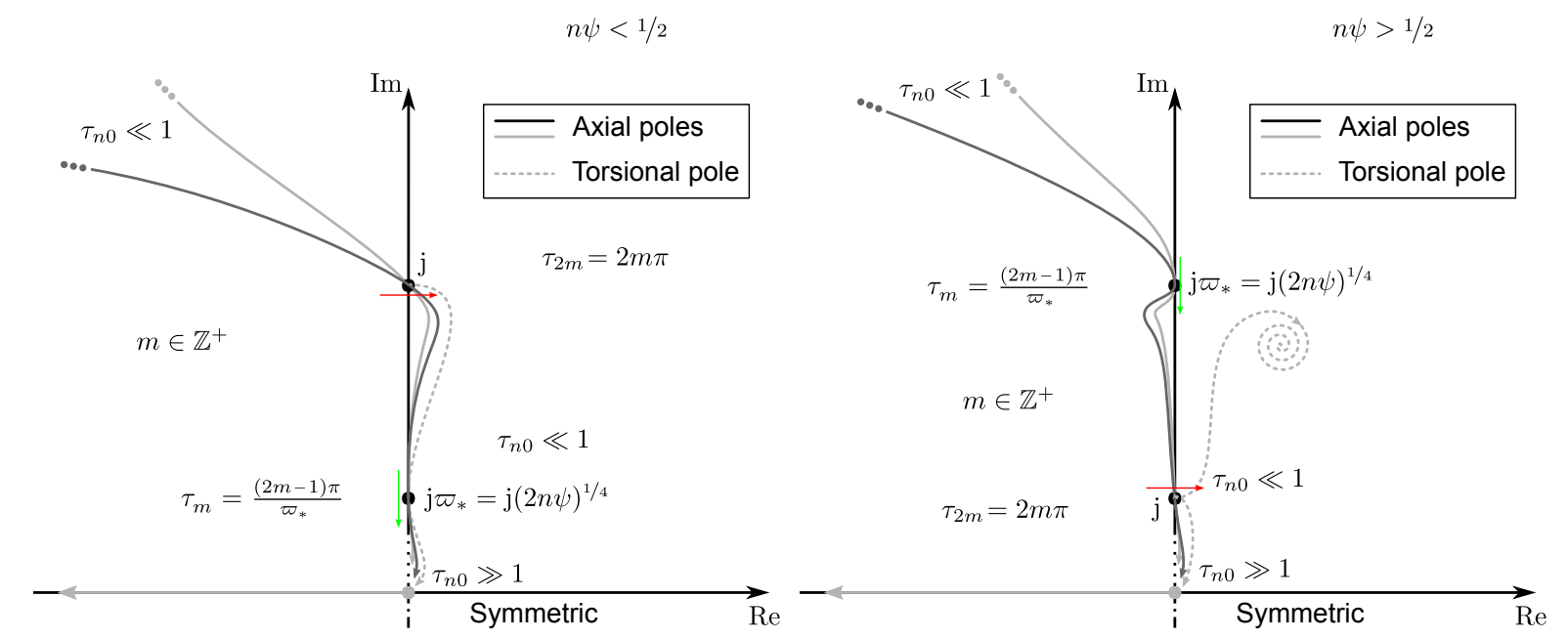

Figure D.4: Root locus of the system poles for parametric configurations with $n \alpha_{0}=n \alpha_{0}^{-}=1 / 2+n \psi-\sqrt{2 n \psi}$. The left figure pertains to range $n \psi<1 / 2$ and the right one to $n \psi>1 / 2$. Only one pair of conjugated crossing points exists, at $s_{c}= \pm \mathrm{j}$, in combination with a tangency point at $s_{c}= \pm \mathrm{j}(2 n \psi)^{1 / 4}$. Depending on the magnitude of $n \psi$, the torsional poles either converge to the origin or to a focus point in the RHS of the complex plane. 